\title{
DISCONTINUOUS GALERKIN APPROXIMATION OF FLOWS IN FRACTURED POROUS MEDIA ON POLYTOPIC GRIDS*
}

\author{
PAOLA F. ANTONIETTI ${ }^{\dagger}$, CHIARA FACCIOLÀ ${ }^{\dagger}$, ALESSANDRO RUSSO $^{\ddagger}$, AND \\ MARCO VERANI ${ }^{\dagger}$
}

\begin{abstract}
We present a numerical approximation of Darcy's flow through a fractured porous medium which employs discontinuous Galerkin methods on polytopic grids. For simplicity, we analyze the case of a single fracture represented by a $(d-1)$-dimensional interface between two $d$-dimensional subdomains, $d=2,3$. We propose a discontinuous Galerkin finite element approximation for the flow in the porous matrix which is coupled with a conforming finite element scheme for the flow in the fracture. Suitable (physically consistent) coupling conditions complete the model. We theoretically analyze the resulting formulation, prove its well-posedness, and derive optimal a priori error estimates in a suitable (mesh-dependent) energy norm. Two-dimensional numerical experiments are reported to assess the theoretical results.
\end{abstract}

Key words. discontinuous Galerkin, polytopic grids, flows in fractured porous media

AMS subject classifications. $65 \mathrm{~N} 12,65 \mathrm{~N} 15$

DOI. $10.1137 / 17 \mathrm{M} 1138194$

1. Introduction. Modeling flows in fractured porous media has received increasing attention in the past decades, being fundamental for addressing many environmental and energy problems, such as water resources management, oil migration tracement, isolation of radioactive waste, and groundwater contamination. In these applications the flow is strongly influenced by the presence of fractures, which can act as preferential paths (when their permeability is higher than that of the surrounding medium), or as barriers for the flow (when they are filled with low permeable material). A fracture is typically characterized by a small aperture compared to both its length and the size of the domain and with a different porous structure than the surrounding medium. The task of effectively modeling the interaction between the system of fractures and the porous matrix is particularly challenging. In the following, let us briefly comment on a popular modeling choice to handle such a problem (see, e.g., $[42,29,6])$, which consists in treating fractures as $(d-1)$-dimensional interfaces between $d$-dimensional porous matrices, $d=2,3$. The development of this kind of reduced model, which can be justified in case of fractures with very small width, has been addressed for single-phase flows in several works; see, e.g., [2, 1, 42, 37]. In this paper we adopt the perspective of the single fracture model described in [42]; see also $[29,6]$. A first version of this model has been introduced in [1] and [2] under the assumption of large permeability in the fracture. In [42] the model has been further

*Submitted to the journal's Methods and Algorithms for Scientific Computing section July 11, 2017; accepted for publication (in revised form) October 25, 2018; published electronically January $2,2019$.

http://www.siam.org/journals/sisc/41-1/M113819.html

Funding: The first and second authors were supported by SIR Project n. RBSI14VT0S "PolyPDEs: Non-conforming polyhedral finite element methods for the approximation of partial differential equations" funded by MIUR. The fourth author was partially supported by the Italian research grant Prin 2012 2012HBLYE4 "Metodologie innovative nella modellistica differenziale numerica." The first, second, and fourth authors have been partially supported by INdAM-GNCS.

${ }^{\dagger}$ Dipartimento di Matematica, Politecnico di Milano, MOX-Laboratory for Modeling and Scientific Computing (paola.antonietti@polimi.it, chiara.facciola@polimi.it, marco.verani@polimi.it).

${ }_{\ddagger}^{\ddagger}$ Dipartimento di Matematica e Applicazioni, Università degli Studi di Milano-Bicocca and IMATI-CNR, Pavia (alessandro.russo@unimib.it).

A109

Copyright $@$ by SIAM. Unauthorized reproduction of this article is prohibited. 
generalized to handle also fractures with low permeability. Here, the flow in the porous medium is assumed to be governed by Darcy's law, and a suitable reduced version of this law is formulated on the surface modeling the fracture. Physically consistent coupling conditions are added (in strong form) to account for the exchange of fluid between the fracture and the porous medium. The extension of such a coupled model to the case of two-phase flows has been addressed in [38] and [41], while a totally immersed fracture has been considered in [3].

Various numerical methods have been employed in the literature for the approximation of the resulting coupled bulk-fracture model. In this respect, one of the main issues is the construction of the computational grid. Roughly speaking, numerical methods can be classified depending on the interaction between the bulk and the fracture meshes: The computational grid can be either conforming (i.e., matching/aligned) or nonconforming (i.e., nonmatching/nonaligned) with the fracture network. In more traditional approaches the bulk meshes are usually chosen to be aligned with the fractures and to be made of simplicial elements. Some examples can be found in $[2,37,42]$, where mixed finite element schemes have been employed for the discretization. However, in realistic cases, the geometrical conformity of the bulk mesh to the fracture can lead to either low-quality elements or very fine grids, and the process of grid generation might become unaffordable from the computational viewpoint, especially in three dimensions. Indeed, porous media are often characterized by complicated geometries (i.e., large networks of fractures), which may also intersect with small angles or be nearly coincident. An alternative strategy consists in the use of nonconforming discretizations, where the fractures are allowed to arbitrarily cut the bulk grid. This allows for the choice of a fairly regular mesh in the bulk. We mention in particular [29, 38], where an approximation employing the extended finite element method (XFEM) has been proposed (see [34] for a reference about XFEM and [36] for a review).

A good compromise with respect to the above issues is represented by methods based on computational meshes consisting of general polytopic elements (polygons in two dimensions and polyhedra in three dimensions). First, a (possibly structured) bulk grid is generated independently of the fracture networks; second, the elements are cut according to the fracture geometry. The above approach leads to a grid that

(i) is aligned with the fracture network;

(ii) contains possibly arbitrarily shaped elements in the surrounding of fractures;

(iii) is regular far from fractures.

Beyond the simplicity of generating the computational grid based on employing the previously described approach, one of the main advantages of polytopal decompositions over standard simplicial grids is that, even on relatively simple geometries, the average number of elements needed to discretize complicated domains is lower $[7,8]$. This advantage becomes even more evident whenever the domain presents complex geometrical features (large number of fractures, fractures intersecting with small angles, etc.) and the bulk grid is chosen to be matching with the interfaces. Recently, a mixed approximation based on the use of conforming polygonal meshes and mimetic finite differences has been explored in [6] and generalized to networks of fractures in [45]. We also mention the framework for treating flows in discrete fracture networks introduced in $[19,20,18]$, based on either XFEM or virtual element methods (see $[16,17]$ ), and in [27], based on the hybrid high-order method (see [33, 32]).

The aim of this paper is to employ discontinuous Galerkin (DG) finite elements on polytopic grids to discretize the coupled bulk-fracture problem stemming from the modeling of flows in fractured porous media. The inherited flexibility of DG 
methods in handling arbitrarily shaped, nonnecessarily matching grids and elementwise variable polynomial orders represents, in fact, the ideal setting to handle such kinds of problems that typically feature a high level of geometrical complexity. DG, methods were first introduced in the early 1970 s (see, e.g., $[43,35,13,49,10]$ ) as a technique to numerically solve partial differential equations. They have been successfully developed and applied to hyperbolic, elliptic, and parabolic problems arising from a wide range of applications: Various examples can be found, for example, in $[14,26,28,21,40,44,31]$. We refer in particular to [11] for a unified presentation and analysis of DG methods for elliptic problems.

More specifically, the choice of DG methods for addressing the problem of the flow in a fractured porous medium arises quite spontaneously in view of the discontinuous nature of the solution at the matrix-fracture interface. However, this is not the only motivation to employ DG methods in this specific context. Indeed, our differential model is based on the primal form of Darcy's equations for both the bulk and the fracture flows, which are coupled with suitable conditions at the interface. These coupling conditions can be naturally formulated using jump and average operators, so that DG methods turn out to be a very natural and powerful tool for efficiently handling the coupling of the two problems, which is indeed naturally embedded in the variational formulation. In this paper we propose a discretization which combines a DG approximation for the problem in the bulk with a conforming finite element approximation in the fracture. The use of conforming finite elements to discretize the equations in the fracture is made just for the sake of simplicity; other discretization techniques can be employed, and our approach is general enough to take into account straightforwardly also such cases. For the DG approximation of the problem in the bulk, we will refer in particular to $[25,23,4,22]$, where an $h p$-version interior penalty DG method is presented for the numerical solution of second-order elliptic partial differential equations on polytopic grids; see also [24] for a review. This method is characterized by a specific choice of the interior penalty parameter, which allows for the use of polytopic meshes made of elements with edges/faces that may be in arbitrary number (potentially unlimited) and whose measure may be arbitrarily small [22]. Clearly, this is naturally well suited to handle complicated networks of fractures. We analyze the resulting method and prove a priori error estimates, which we numerically test in a two-dimensional setting.

The paper is structured as follows. In section 2 we introduce the governing equations for the coupled problem. The problem is then written in a weak form in section 3 , where we also prove its well-posedness. In section 4 we introduce the DG discretization on polytopic grids of the coupled problem. The main results in the analysis of the method are included in section 5, where we state Theorem 5.2 about well-posedness and Theorem 5.5 containing an a priori error estimate in a suitable (mesh-dependent) norm. Section 6 is devoted to the presentation of a series of two-dimensional numerical experiments assessing both the validity of the theoretical error estimates and the capability of the method of handling more complicated cases, including networks of partially immersed fractures and networks of intersecting fractures. Finally, section 7 contains the proofs and technical details omitted from section 5 .

2. Model problem. Throughout the paper we will employ the following notation. For an open, bounded domain $D \subset \mathbb{R}^{d}, d=2,3$, we denote by $H^{s}(D)$ the standard Sobolev space of order $s$ for a real number $s \geq 0$. For $s=0$, we write $L^{2}(D)$ in place of $H^{0}(D)$. The usual norm on $H^{s}(D)$ is denoted by $\|\cdot\|_{H^{s}(D)}$ and the usual seminorm by $|\cdot|_{H^{s}(D)}$. Furthermore, we will denote by $\mathbb{P}_{k}(D)$ the space of polynomials of total degree less than or equal to $k \geq 1$ on $D$. The symbol $\lesssim$ (and $\gtrsim$ ) will signify 


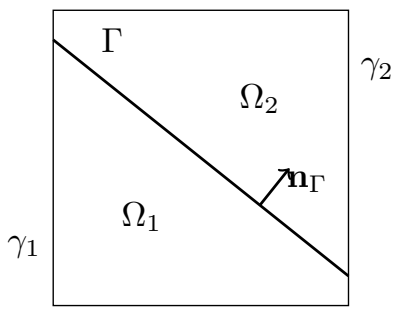

FIG. 1. The subdomains $\Omega_{1}$ and $\Omega_{2}$ separated by the fracture $\Gamma$ considered as an interface.

that the inequalities hold up to multiplicative constants which are independent of the discretization parameters but might depend on the physical parameters.

In the following we present the governing equations for our model, which is a variant of the model derived in [42], where the coupling conditions are imposed in a weak sense. The flow of an incompressible fluid through a fractured $d$-dimensional porous medium, $d=2,3$, can be described by the following three ingredients:

1. the governing equations for the flow in the porous medium;

2. the governing equations for the flow in the fractures;

3. a set of physically consistent conditions which couple the problems in the bulk and fractures along their interfaces.

For simplicity, we will assume that there is only one fracture in the porous medium and that the fracture cuts the domain exactly into two disjoint connected subregions (see Figure 1 for a two-dimensional example), following the approach of [6] and [29]. The extension to a network of disjoint fractures can be treated analogously, while the case of an immersed fracture is more complex to analyze [3] and will be the subject of future research. Nevertheless, the capability of our method to deal with networks of partially immersed fractures and with networks of intersecting fractures will be explored via numerical experiments in section 6.5. Let $\Omega \subset \mathbb{R}^{d}, d=2,3$, be an open, bounded, convex polygonal/polyhedral domain representing the porous matrix. We suppose that the fracture is a $(d-1)$-dimensional $\mathcal{C}^{\infty}$ manifold with no curvature $\Gamma \subset \mathbb{R}^{d-1}, d=2,3$, whose measure satisfies $|\Gamma|=\mathcal{O}(1)$, and assume that $\Gamma$ separates $\Omega$ into two connected subdomains, which are disjoint, i.e., $\Omega \backslash \Gamma=\Omega_{1} \cup \Omega_{2}$ with $\Omega_{1} \cap \Omega_{2}=\emptyset$. For $i=1,2$, we denote by $\gamma_{i}$ the part of boundary of $\Omega_{i}$ shared with the boundary of $\Omega$, i.e., $\gamma_{i}=\partial \Omega_{i} \cap \partial \Omega$. We denote by $\mathbf{n}_{i}, i=1,2$ the unit normal vector to $\Gamma$ pointing outwards from $\Omega_{i}$, and for a (regular enough) scalar-valued function $v$ and a (regular enough) vector-valued function $\tau$, we define the standard jump and average operators across $\Gamma$ as

$$
\begin{aligned}
\{v\}=\frac{1}{2}\left(v_{1}+v_{2}\right) & \llbracket v \rrbracket=v_{1} \mathbf{n}_{1}+v_{2} \mathbf{n}_{2}, \\
\{\boldsymbol{\tau}\}=\frac{1}{2}\left(\boldsymbol{\tau}_{1}+\boldsymbol{\tau}_{2}\right) & \llbracket \boldsymbol{\tau} \rrbracket=\boldsymbol{\tau}_{1} \cdot \mathbf{n}_{1}+\boldsymbol{\tau}_{2} \cdot \mathbf{n}_{2},
\end{aligned}
$$

where the subscript $i=1,2$ denotes the restriction to the subdomain $\Omega_{i}$. Moreover, we denote by $\mathbf{n}_{\Gamma}$ the normal unit vector on $\Gamma$ with a fixed orientation from $\Omega_{1}$ to $\Omega_{2}$, so that we have $\mathbf{n}_{\Gamma}=\mathbf{n}_{1}=-\mathbf{n}_{2}$.

2.1. Governing equations. According to the above discussion, we suppose that the flow in the bulk is governed by Darcy's law. Let $\boldsymbol{\nu}=\boldsymbol{\nu}(x) \in \mathbb{R}^{d \times d}$ be the bulk permeability tensor, which satisfies the following regularity assumptions: 
(i) $\boldsymbol{\nu}$ is a symmetric, positive definite tensor whose entries are bounded, piecewise continuous real-valued functions;

(ii) $\boldsymbol{\nu}$ is uniformly bounded from below and above, i.e., $\mathbf{x}^{T} \mathbf{x} \lesssim \mathbf{x}^{T} \boldsymbol{\nu} \mathbf{x} \lesssim \mathbf{x}^{T} \mathbf{x}$ $\forall \mathbf{x} \in \mathbb{R}^{d}$.

Given a function $f \in L^{2}(\Omega)$ representing a source term and $g \in H^{1 / 2}(\partial \Omega)$, the motion of an incompressible fluid in each domain $\Omega_{i}, i=1,2$ with pressure $p_{i}$ is described by:

$$
\begin{aligned}
-\nabla \cdot\left(\boldsymbol{\nu}_{i} \nabla p_{i}\right) & =f_{i} & & \text { in } \Omega_{i}, \quad i=1,2, \\
p_{i} & =g_{i} & & \text { on } \gamma_{i}, \quad i=1,2 .
\end{aligned}
$$

Here we have denoted by $\boldsymbol{\nu}_{i}$ and $f_{i}$ the restrictions of $\boldsymbol{\nu}$ and $f$ to $\Omega_{i}, i=1,2$, respectively, and by $g_{i}$ the restriction of $g$ to $\gamma_{i}, i=1,2$ (for simplicity, we have imposed Dirichlet boundary conditions on both $\gamma_{1}$ and $\gamma_{2}$ ).

The second ingredient for the model is represented by the governing equations for the fracture flow. In our model the fracture is treated as a $(d-1)$-dimensional manifold immersed in a $d$-dimensional object. If we assume that the fractures are filled by a porous medium with different porosity and permeability than the surroundings, Darcy's law can be used also for modeling the flow along the fractures [15]. The reduced model is then obtained through a process of averaging across the fracture: In the beginning the fracture is treated as a $d$-dimensional subdomain of $\Omega$ that separates it into two disjoint subdomains. Then Darcy's equations are written on the fracture in the normal and tangential components, and the tangential component is integrated along the thickness $\ell_{\Gamma}>0$ of the fracture domain, which is typically some orders of magnitude smaller than the size of the domain. We refer to [42] for a rigorous derivation of the reduced mathematical model. Note that in [42] this averaging process is carried out for the flow equations written in mixed form. Here, we consider the corresponding model in primal form.

The fracture flow is then characterized by the fracture permeability tensor $\boldsymbol{\nu}_{\Gamma}$, which is assumed to satisfy the same regularity assumptions as those satisfied by the bulk permeability $\boldsymbol{\nu}$ and to have a block-diagonal structure of the form

$$
\boldsymbol{\nu}_{\Gamma}=\left[\begin{array}{cc}
\nu_{\Gamma}^{n} & 0 \\
0 & \boldsymbol{\nu}_{\Gamma}^{\tau}
\end{array}\right]
$$

when written in its normal and tangential components. Here, $\boldsymbol{\nu}_{\Gamma}^{\tau} \in \mathbb{R}^{(d-1) \times(d-1)}$ is a positive definite, uniformly bounded tensor (it reduces to a positive number for $d=2$ ) representing the tangential component of the permeability of the fracture.

Setting $\partial \Gamma=\Gamma \cap \partial \Omega$ and denoting by $p_{\Gamma}$ the fracture pressure, the governing equations for the fracture flow read as

$$
\begin{array}{rlr}
-\nabla_{\tau} \cdot\left(\boldsymbol{\nu}_{\Gamma}^{\tau} \ell_{\Gamma} \nabla_{\tau} p_{\Gamma}\right) & =f_{\Gamma}+\llbracket-\boldsymbol{\nu} \nabla p \rrbracket & \text { in } \Gamma, \\
p_{\Gamma} & =g_{\Gamma} & \text { on } \partial \Gamma,
\end{array}
$$

where $f_{\Gamma} \in L^{2}(\Gamma), g_{\Gamma} \in H^{1 / 2}(\partial \Gamma)$ and $\nabla_{\tau}$ and $\nabla_{\tau}$. denote the tangential gradient and divergence operators, respectively. Equation (4a) represents Darcy's law in the direction tangential to the fracture, where a source term $\llbracket-\boldsymbol{\nu} \nabla p \rrbracket$ is introduced to take into account the contribution of the subdomain flows to the fracture flow [42]. For the sake of simplicity, we impose Dirichlet boundary conditions at the boundary $\partial \Gamma$ of the fracture $\Gamma$. 
Finally, following [42], we provide the interface conditions to couple problems $(2 \mathrm{a})-(2 \mathrm{~b})$ and $(4 \mathrm{a})-(4 \mathrm{~b})$. Let $\xi$ be a positive real number, $\xi \neq \frac{1}{2}$, that will be chosen later on. The coupling conditions are given by

$$
\begin{aligned}
-\{\boldsymbol{\nu} \nabla p\} \cdot \mathbf{n}_{\Gamma} & =\beta_{\Gamma}\left(p_{1}-p_{2}\right) & & \text { on } \Gamma, \\
-\llbracket \boldsymbol{\nu} \nabla p \rrbracket & =\alpha_{\Gamma}\left(\{p\}-p_{\Gamma}\right) & & \text { on } \Gamma,
\end{aligned}
$$

where

$$
\beta_{\Gamma}=\frac{1}{2 \eta_{\Gamma}}, \quad \alpha_{\Gamma}=\frac{2}{\eta_{\Gamma}(2 \xi-1)},
$$

and $\eta_{\Gamma}=\frac{\ell_{\Gamma}}{\nu_{\Gamma}^{n}}, \boldsymbol{\nu}_{\Gamma}^{n}$ being the normal component of the fracture permeability tensor; see (3). Note that the coupling conditions are formulated employing jump and average operators. This turns out to be convenient for employing DG methods in the discretization.

In conclusion, the coupled model problem reads as

$$
\begin{aligned}
& -\nabla \cdot\left(\boldsymbol{\nu}_{i} \nabla p_{i}\right)=f_{i} \quad \text { in } \Omega_{i}, \quad i=1,2, \\
& p_{i}=g_{i} \quad \text { on } \gamma_{i}, \quad i=1,2, \\
& -\nabla_{\tau} \cdot\left(\boldsymbol{\nu}_{\Gamma}^{\tau} \ell_{\Gamma} \nabla_{\tau} p_{\Gamma}\right)=f_{\Gamma}+\llbracket-\boldsymbol{\nu} \nabla p \rrbracket \quad \text { in } \Gamma \text {, } \\
& p_{\Gamma}=g_{\Gamma} \quad \text { on } \partial \Gamma, \\
& -\{\boldsymbol{\nu} \nabla p\} \cdot \mathbf{n}_{\Gamma}=\beta_{\Gamma}\left(p_{1}-p_{2}\right) \quad \text { on } \Gamma, \\
& -\llbracket \nu \nabla p \rrbracket=\alpha_{\Gamma}\left(\{p\}-p_{\Gamma}\right) \quad \text { on } \Gamma \text {. }
\end{aligned}
$$

Note that the introduction of the parameter $\xi$ yields a family of models; see [42] for more details.

3. Weak formulation and its well-posedness. In this section we present a weak formulation of our model problem (7) where the coupling conditions (5a)-(5b) are imposed in a weak sense and prove its well-posedness. For the sake of simplicity we will assume that homogeneous Dirichlet boundary conditions are imposed for both the bulk and the fracture problems, i.e., $g_{i}=0, i=1,2$, and $g_{\Gamma}=0$. The extension to the general nonhomogeneous case is straightforward. We introduce the following spaces:

$$
V^{b}=\left\{p=\left(p_{1}, p_{2}\right) \in V_{1}^{b} \times V_{2}^{b}\right\}, \quad V^{\Gamma}=H_{0}^{1}(\Gamma) \cap H^{s}(\Gamma),
$$

where we define, for $i=1,2$ and $s \geq 1, V_{i}^{b}=H^{s}\left(\Omega_{i}\right) \cap H_{0, \gamma_{i}}^{1}\left(\Omega_{i}\right)$, with $H_{0, \gamma_{i}}^{1}\left(\Omega_{i}\right)=$ $\left\{q \in H^{1}\left(\Omega_{i}\right)\right.$ s.t. $\left.\left.q\right|_{\gamma_{i}}=0\right\}$.

Next we introduce the bilinear forms $\mathcal{A}_{b}: V^{b} \times V^{b} \rightarrow \mathbb{R}, \mathcal{A}_{\Gamma}: V^{\Gamma} \times V^{\Gamma} \rightarrow \mathbb{R}$ and $\mathcal{I}:\left(V^{b} \times V^{\Gamma}\right) \times\left(V^{b} \times V^{\Gamma}\right) \rightarrow \mathbb{R}$ defined as

$$
\begin{aligned}
\mathcal{A}_{b}(p, q)=\sum_{i=1}^{2} \int_{\Omega_{i}} \boldsymbol{\nu}_{i} \nabla p_{i} \cdot \nabla q_{i}, \quad \mathcal{A}_{\Gamma}\left(p_{\Gamma}, q_{\Gamma}\right)=\int_{\Gamma} \boldsymbol{\nu}_{\Gamma}^{\tau} \ell_{\Gamma} \nabla_{\tau} p_{\Gamma} \cdot \nabla_{\tau} q_{\Gamma}, \\
\mathcal{I}\left(\left(p, p_{\Gamma}\right),\left(q, q_{\Gamma}\right)\right)=\int_{\Gamma} \beta_{\Gamma} \llbracket p \rrbracket \cdot \llbracket q \rrbracket+\int_{\Gamma} \alpha_{\Gamma}\left(\{p\}-p_{\Gamma}\right)\left(\{q\}-q_{\Gamma}\right),
\end{aligned}
$$

Copyright $@$ ㅇ by SIAM. Unauthorized reproduction of this article is prohibited. 
where $\alpha_{\Gamma}$ and $\beta_{\Gamma}$ are defined as in (6). Clearly, the bilinear forms $\mathcal{A}_{b}(\cdot, \cdot)$ and $\mathcal{A}_{\Gamma}(\cdot, \cdot)$ take into account the problems in the bulk and in the fracture, respectively, while $\mathcal{I}(\cdot, \cdot)$ takes into account the interface conditions (5). We also introduce the linear functional $\mathcal{L}_{b}: V^{b} \rightarrow \mathbb{R}$ defined as $\mathcal{L}_{b}(q)=\sum_{i=1}^{2} \int_{\Omega_{i}} f q_{i}$ and the linear functional $\mathcal{L}_{\Gamma}: V^{\Gamma} \rightarrow \mathbb{R}$ defined as $\mathcal{L}_{\Gamma}\left(q_{\Gamma}\right)=\int_{\Gamma} f_{\Gamma} q_{\Gamma}$, which represent the source terms in the bulk and the fracture, respectively.

With the above notation, the weak formulation of our model problem reads as follows. Find $\left(p, p_{\Gamma}\right) \in V^{b} \times V^{\Gamma}$ such that for all $\left(q, q_{\Gamma}\right) \in V^{b} \times V^{\Gamma}$,

$$
\mathcal{A}\left(\left(p, p_{\Gamma}\right),\left(q, q_{\Gamma}\right)\right)=\mathcal{L}\left(q, q_{\Gamma}\right),
$$

where $\mathcal{A}:\left(V^{b} \times V^{\Gamma}\right) \times\left(V^{b} \times V^{\Gamma}\right) \rightarrow \mathbb{R}$ is defined as the sum of the bilinear forms just introduced,

$$
\mathcal{A}\left(\left(p, p_{\Gamma}\right),\left(q, q_{\Gamma}\right)\right)=\mathcal{A}_{b}(p, q)+\mathcal{A}_{\Gamma}\left(p_{\Gamma}, q_{\Gamma}\right)+\mathcal{I}\left(\left(p, p_{\Gamma}\right),\left(q, q_{\Gamma}\right)\right),
$$

and the linear operator $\mathcal{L}: V^{b} \times V^{\Gamma} \rightarrow \mathbb{R}$ is defined as

$$
\mathcal{L}\left(q, q_{\Gamma}\right)=\mathcal{L}_{b}(q)+\mathcal{L}_{\Gamma}\left(q_{\Gamma}\right) .
$$

Next, we show that formulation (9) is well-posed. To this aim we introduce the following norm on $V^{b} \times V^{\Gamma}$ :

$$
\begin{aligned}
\left\|\left(q, q_{\Gamma}\right)\right\|_{\mathcal{E}}^{2}=\sum_{i=1}^{2}\left\|\boldsymbol{\nu}_{i}^{1 / 2} \nabla q_{i}\right\|_{L^{2}\left(\Omega_{i}\right)}^{2} & +\left\|\left(\boldsymbol{\nu}_{\Gamma}^{\tau} \ell_{\Gamma}\right)^{1 / 2} \nabla_{\tau} q_{\Gamma}\right\|_{L^{2}(\Gamma)}^{2} \\
& +\left\|\beta_{\Gamma}^{1 / 2} \llbracket q \rrbracket\right\|_{L^{2}(\Gamma)}^{2}+\left\|\alpha_{\Gamma}^{1 / 2}\left(\{q\}-q_{\Gamma}\right)\right\|_{L^{2}(\Gamma)}^{2} .
\end{aligned}
$$

This is clearly a norm if $\alpha_{\Gamma} \geq 0$. Since $\alpha_{\Gamma}=\frac{2}{\eta_{\Gamma}(2 \xi-1)}$ (see (6)), from now on, we will assume that $\xi>1 / 2$. We remark that the same condition on the parameter $\xi$ has been found also in [6] and [42].

Theorem 3.1. Let $\xi>1 / 2$. Then problem (9) is well-posed.

Proof. We show that $\mathcal{A}(\cdot, \cdot)$ is continuous and coercive on $V^{b} \times V^{\Gamma}$ equipped with the norm (12), as well as $\mathcal{L}(\cdot)$ is continuous on $V^{b} \times V^{\Gamma}$ with respect to the same norm. Then the existence and uniqueness of the solution, as well as linear dependence on the data, follow directly from Lax-Milgram's lemma. Coercivity is straightforward, as we clearly have that $\mathcal{A}\left(\left(q, q_{\Gamma}\right),\left(q, q_{\Gamma}\right)\right)=\left\|\left(q, q_{\Gamma}\right)\right\|_{\mathcal{E}}^{2}$ for any $\left(q, q_{\Gamma}\right) \in V^{b} \times V^{\Gamma}$. On the other hand, continuity is a direct consequence of the Cauchy-Schwarz inequality, while continuity of $\mathcal{L}(\cdot)$ on $V^{b} \times V^{\Gamma}$ is guaranteed by the regularity of the forcing term $f$.

4. Numerical discretization. In this section we present a numerical discretization of our problem which combines a DG approximation on general polytopic elements for the problem in the bulk, with a conforming finite element approximation in the fracture (see Remark 4.2 below). DG methods are very convenient for handling the discontinuity of the bulk pressure across the fracture as well as the coupling of the bulk-fracture problems, which has been formulated using jump and average operators. As a result, we can employ the tools offered by DG methods to prove the well-posedness of our discrete method (see Proposition 5.2, below). In particular, we 
will adopt the techniques developed in $[25,23,4,22]$, where an $h p$-version interior penalty DG method for the numerical solution of elliptic problems on polytopic meshes has been proposed and analyzed. This method is characterized by a specific choice of the interior penalty parameter, which allows for face-degeneration. In $[25,23,4]$ it is assumed that the number of edges/faces of each mesh element is uniformly bounded. In $[22,9]$, this assumption is no longer required (i.e., elements with an arbitrary number of possibly degenerating faces/edges are admitted). However, this comes at the cost of adding an assumption (see 7.1 below) that may be regarded as the natural generalization to polytopic grids of the classical shape-regularity assumption [24]. Here, we work in the setting of $[22,9]$.

We start with the introduction of some useful notation. We consider a family of meshes $\mathcal{T}_{h}$ made of disjoint open polygonal/polyhedral elements which are aligned with the fracture $\Gamma$, so that any element $E \in \mathcal{T}_{h}$ cannot be cut by $\Gamma$. Note that, since $\Omega_{1}$ and $\Omega_{2}$ are disjoint, each element $E$ belongs exactly to one of the two subdomains. In order to admit hanging nodes, following $[25,23,4]$, we introduce the concept of mesh interfaces, which are defined to be the intersection of the $(d-1)$-dimensional facets of neighboring elements. In the case when $d=2$, the interfaces of an element $E \in \mathcal{T}_{h}$ simply consists of line segments. For $d=3$, we assume that it is possible to subdivide each interface into a set of coplanar triangles. We then use the terminology "face" (or edge) to refer to a $(d-1)$-dimensional simplex (line segment for $d=2$ or triangle for $d=3$ ), which forms part of the interface of an element. Note that for $d=2$, face and interface of an element $E \in \mathcal{T}_{h}$ coincide. Following [25, 23, 4], for $d=3$ we assume that, for each mesh interface, a subtriangulation into faces is provided. Notice that no limitation is imposed on either the number of faces of each polygon $E \in \mathcal{T}_{h}$ or the relative size of element faces compared to its diameter.

Clearly each mesh $\mathcal{T}_{h}$ induces a subdivision of the fracture $\Gamma$ into faces that we will denote by $\Gamma_{h}$. Moreover, we denote by $\mathcal{F}_{h}$ the set of all open interfaces of the decomposition $\mathcal{T}_{h}$ if $d=2$ and the union of all open triangles belonging to the subtriangulation of all mesh interfaces if $d=3$ (so that $\mathcal{F}_{h}$ is always defined as a set of $(d-1)$-dimensional simplices). Moreover, we write $\mathcal{F}_{h}=\mathcal{F}_{h}^{I} \cup \mathcal{F}_{h}^{B} \cup \Gamma_{h}$, where $\mathcal{F}_{h}^{B}$ is the set of boundary faces and $\mathcal{F}_{h}^{I}$ is the set of interior faces not belonging to the fracture. For each element $E \in \mathcal{T}_{h}$, we denote by $|E|$ its measure and by $h_{E}$ its diameter, and we set $h=\max _{E \in \mathcal{T}_{h}} h_{E}$. Finally, given an element $E \in \mathcal{T}_{h}$, for any face/edge $F \subset \partial E$ we define $\mathbf{n}_{F}$ as the unit normal vector on $F$ that points outside $E$. We can then define the standard (see [11]) jump and average operators across an edge $F \in \mathcal{F}_{h}$ for (regular enough) scalar and vector-valued functions similarly to (1).

Given a partition $\mathcal{T}_{h}$ of the domain, we denote by $H^{s}\left(\mathcal{T}_{h}\right), s \geq 1$, the standard broken Sobolev space.

With the aim of building a DG-conforming finite element approximation, we choose to set the discrete problem in the finite-dimensional spaces:

$$
\begin{aligned}
V_{h}^{b} & =\left\{q_{h} \in L^{2}(\Omega):\left.q_{h}\right|_{E} \in \mathbb{P}_{k_{E}}(E) \forall E \in \mathcal{T}_{h}\right\}, & k_{E} \geq 1 \quad \forall E \in \mathcal{T}_{h} \\
V_{h}^{\Gamma} & =\left\{q_{h}^{\Gamma} \in \mathcal{C}^{0}(\Gamma):\left.q_{h}^{\Gamma}\right|_{F} \in \mathbb{P}_{k}(F) \forall F \in \Gamma_{h}\right\} & k \geq 1 .
\end{aligned}
$$

Note that to each element $E \in \mathcal{T}_{h}$ is associated the polynomial degree $k_{E}$. We also remark that the polynomial degrees in the bulk and fracture discrete spaces just defined are chosen independently. 
Next, we introduce the bilinear forms $\mathcal{A}_{b}^{D G}: V_{h}^{b} \times V_{h}^{b} \rightarrow \mathbb{R}$ and $\mathcal{I}^{D G}:\left(V_{h}^{b} \times V_{h}^{\Gamma}\right) \times$ $\left(V_{b}^{h} \times V_{h}^{\Gamma}\right) \rightarrow \mathbb{R}$, defined as follows:

$$
\begin{aligned}
\mathcal{A}_{b}^{D G}\left(p_{h}, q_{h}\right)= & \sum_{E \in \mathcal{T}_{h}} \int_{E} \boldsymbol{\nu} \nabla p_{h} \cdot \nabla q_{h}-\sum_{F \in \mathcal{F}_{h} \backslash \Gamma_{h}} \int_{F}\left\{\boldsymbol{\nu} \nabla p_{h}\right\} \cdot \llbracket q_{h} \rrbracket \\
& -\sum_{F \in \mathcal{F}_{h} \backslash \Gamma_{h}} \int_{F}\left\{\boldsymbol{\nu} \nabla q_{h}\right\} \cdot \llbracket p_{h} \rrbracket+\sum_{F \in \mathcal{F}_{h} \backslash \Gamma_{h}} \int_{F} \sigma_{F} \llbracket p_{h} \rrbracket \cdot \llbracket q_{h} \rrbracket, \\
\mathcal{I}^{D G}\left(\left(p_{h}, p_{h}^{\Gamma}\right),\left(q_{h}, q_{h}^{\Gamma}\right)\right) & =\sum_{F \in \Gamma_{h}} \int_{F} \beta_{\Gamma} \llbracket p_{h} \rrbracket \cdot \llbracket q_{h} \rrbracket+\sum_{F \in \Gamma_{h}} \int_{F} \alpha_{\Gamma}\left(\left\{p_{h}\right\}-p_{h}^{\Gamma}\right)\left(\left\{q_{h}\right\}-q_{h}^{\Gamma}\right) .
\end{aligned}
$$

The nonnegative function $\sigma \in L^{\infty}\left(\mathcal{F}_{h} \backslash \Gamma_{h}\right)$ is the discontinuity penalization parameter $\left(\sigma_{F}=\left.\sigma\right|_{F}\right.$ for $\left.F \in \mathcal{F}_{h} \backslash \Gamma_{h}\right)$. The precise definition of $\sigma$ will be presented in Definition 5.1 below. Finally we define the linear functional $\mathcal{L}_{b}^{D G}: V_{h}^{b} \rightarrow \mathbb{R}$ as

$$
\mathcal{L}_{b}^{D G}\left(q_{h}\right)=\sum_{E \in \mathcal{T}_{h}} \int_{E} f q_{h}
$$

Remark 4.1. Since we are imposing homogeneous boundary conditions, $\mathcal{L}_{b}^{D G}$ has the same structure of the linear functional $\mathcal{L}_{b}$ previously defined. In general, for $g \neq 0$, $\mathcal{L}_{b}^{D G}$ contains some additional terms:

$$
\mathcal{L}_{b}^{D G}\left(q_{h}\right)=\sum_{E \in \mathcal{T}_{h}} \int_{E} f q_{h}+\sum_{F \in \mathcal{F}_{h}^{B}} \int_{F}\left(-\boldsymbol{\nu} \nabla q_{h} \cdot \mathbf{n}_{F}+\sigma_{F} q_{h}\right) g .
$$

The DG discretization of problem (9) reads as follows. Find $\left(p_{h}, p_{h}^{\Gamma}\right) \in V_{h}^{b} \times V_{h}^{\Gamma}$ such that

$$
\mathcal{A}_{h}\left(\left(p_{h}, p_{h}^{\Gamma}\right),\left(q_{h}, q_{h}^{\Gamma}\right)\right)=\mathcal{L}_{h}\left(q_{h}, q_{h}^{\Gamma}\right) \quad \forall\left(q_{h}, q_{h}^{\Gamma}\right) \in V_{h}^{b} \times V_{h}^{\Gamma},
$$

where $\mathcal{A}_{h}:\left(V_{h}^{b} \times V_{h}^{\Gamma}\right) \times\left(V_{h}^{b} \times V_{h}^{\Gamma}\right) \rightarrow \mathbb{R}$ is defined as

$$
\mathcal{A}_{h}\left(\left(p_{h}, p_{h}^{\Gamma}\right),\left(q_{h}, q_{h}^{\Gamma}\right)\right)=\mathcal{A}_{b}^{D G}\left(p_{h}, q_{h}\right)+\mathcal{A}_{\Gamma}\left(p_{h}^{\Gamma}, q_{h}^{\Gamma}\right)+\mathcal{I}^{D G}\left(\left(p_{h}, p_{h}^{\Gamma}\right),\left(q_{h}, q_{h}^{\Gamma}\right)\right)
$$

and $\mathcal{L}_{h}: V_{h}^{b} \times V_{h}^{\Gamma} \rightarrow \mathbb{R}$ is defined as

$$
\mathcal{L}_{h}\left(q_{h}, q_{h}^{\Gamma}\right)=\mathcal{L}_{b}^{D G}\left(q_{h}\right)+\mathcal{L}_{\Gamma}\left(q_{h}^{\Gamma}\right) .
$$

Note that the discrete bilinear form $\mathcal{A}_{h}$ has the same structure as the bilinear form $\mathcal{A}$ previously defined, being the sum of three different components, each representing a specific part of the problem.

Remark 4.2. The choice of employing a conforming finite element approximation for the flow in the fracture has been made only in order to keep the analysis of the numerical method as clear as possible; cf. section 7 . In this case, we need to make an additional assumption for the fracture mesh $\Gamma_{h}$ to be made of shape-regular triangles. However, we remark that the use of DG methods for the fracture problem as well would make this assumption unnecessary, thus allowing for the use of very general meshes. If DG methods are employed for the fracture as well, the discrete bilinear form for the fracture problem becomes 


$$
\begin{aligned}
\mathcal{A}_{\Gamma}^{D G}\left(p_{h}^{\Gamma}, q_{h}^{\Gamma}\right)=\int_{\Gamma_{h}} \boldsymbol{\nu}_{\Gamma}^{\tau} \ell_{\Gamma} \nabla_{\tau} p_{h}^{\Gamma} \cdot \nabla_{\tau} q_{h}^{\Gamma}-\sum_{e \in \mathcal{E}_{\Gamma, h}} \int_{e}\left\{\boldsymbol{\nu}_{\Gamma}^{\tau} \ell_{\Gamma} \nabla_{\tau} p_{h}^{\Gamma}\right\} \cdot \llbracket q_{h}^{\Gamma} \rrbracket \\
-\sum_{e \in \mathcal{E}_{\Gamma, h}} \int_{e}\left\{\boldsymbol{\nu}_{\Gamma}^{\tau} \ell_{\Gamma} \nabla_{\tau} q_{h}^{\Gamma}\right\} \cdot \llbracket p_{h}^{\Gamma} \rrbracket+\sum_{e \in \mathcal{E}_{\Gamma, h}} \int_{e} \sigma_{e} \llbracket p_{h}^{\Gamma} \rrbracket \cdot \llbracket q_{h}^{\Gamma} \rrbracket,
\end{aligned}
$$

where $\mathcal{E}_{\Gamma, h}$ is the set of the fracture edges. Since the analysis of the numerical method with a DG discretization for both the bulk and the fracture problems would require additional technicalities, without giving any deeper understanding of the problem, we omit it and only report the main convergence result (see Remark 5.6 below).

5. Well-posedness and approximation. In this section we state the main results about stability and error analysis of formulation (13). We leave the details of the proofs to section 7 .

For simplicity, we suppose that the permeability tensors $\boldsymbol{\nu}$ and $\boldsymbol{\nu}_{\Gamma}$ are piecewise constant on mesh elements, i.e., $\left.\boldsymbol{\nu}\right|_{E} \in\left[\mathbb{P}_{0}(E)\right]^{d \times d}$ for all $E \in \mathcal{T}_{h}$ and $\left.\boldsymbol{\nu}_{\Gamma}\right|_{F} \in$ $\left[\mathbb{P}^{0}(F)\right]^{(d-1) \times(d-1)}$ for all $F \in \Gamma_{h}$. In the following, we will employ the notation $\overline{\boldsymbol{\nu}}_{E}=\left.|\sqrt{\boldsymbol{\nu}}|_{E}\right|_{2} ^{2}$, where $|\cdot|_{2}$ denotes the $l_{2}$-norm.

To complete the definition of our method, we need to specify the form of the discontinuity penalization parameter $\sigma$. Taking as a reference $[25,23,4,22]$, we give the following.

Definition 5.1. The discontinuity-penalization parameter $\sigma: \mathcal{F}_{h} \backslash \Gamma_{h} \rightarrow \mathbb{R}^{+}$is defined facewise by

$$
\sigma(\boldsymbol{x})=\sigma_{0} \begin{cases}\max _{E \in\left\{E^{+}, E^{-}\right\}} \frac{\overline{\boldsymbol{\nu}}_{E}\left(k_{E}+1\right)\left(k_{E}+d\right)}{h_{E}} & \text { if } \boldsymbol{x} \in F \in \mathcal{F}_{h}^{I}, \bar{F}=\partial \bar{E}^{+} \cap \partial \bar{E}^{-} \\ \frac{\overline{\boldsymbol{\nu}}_{E}\left(k_{E}+1\right)\left(k_{E}+d\right)}{h_{E}} & \text { if } \boldsymbol{x} \in F \in \mathcal{F}_{h}^{B}, \bar{F}=\partial \bar{E} \cap \partial \bar{\Omega},\end{cases}
$$

with $\sigma_{0}>0$ independent of $k_{E},|E|$, and $|F|$.

We can then state the following stability result.

Proposition 5.2. Let $\sigma$ be defined as is (17). Then, if $\sigma_{0}$ is chosen sufficiently large, problem (13) is well-posed.

Proof. See section 7 .

Next, we provide an a priori error estimate for problem (13), proving that the discrete solution $\left(p_{h}, p_{h}^{\Gamma}\right)$ converges to the exact solution $\left(p, p_{\Gamma}\right)$. To this aim, we equip the space $V^{b}(h)=V_{h}^{b}+V^{b}$ with the following norm:

$$
\left\|\left(q, q_{h}^{\Gamma}\right)\right\|_{\mathcal{E}_{h}}^{2}=\|q\|_{D G}^{2}+\left\|q_{h}^{\Gamma}\right\|_{\Gamma}^{2}+\left\|\left(q, q_{h}^{\Gamma}\right)\right\|_{\mathcal{I}}^{2},
$$

where

$$
\begin{aligned}
\|q\|_{D G}^{2} & =\sum_{E \in \mathcal{T}_{h}}\left\|\boldsymbol{\nu}^{1 / 2} \nabla q\right\|_{L^{2}(E)}^{2}+\sum_{F \in \mathcal{F}_{h} \backslash \Gamma_{h}}\left\|\sigma_{F}^{1 / 2} \llbracket q \rrbracket\right\|_{L^{2}(F)}^{2}, \\
\left\|q_{h}^{\Gamma}\right\|_{\Gamma}^{2} & =\sum_{F \in \Gamma_{h}}\left\|\left(\boldsymbol{\nu}_{\Gamma}^{\tau} \ell_{\Gamma}\right)^{1 / 2} \nabla_{\tau} q_{h}^{\Gamma}\right\|_{L^{2}(F)}^{2}, \\
\left\|\left(q, q_{h}^{\Gamma}\right)\right\|_{\mathcal{I}}^{2} & =\sum_{F \in \Gamma_{h}}\left\|\beta_{\Gamma}^{1 / 2} \llbracket q \rrbracket\right\|_{L^{2}(F)}^{2}+\sum_{F \in \Gamma_{h}}\left\|\alpha_{\Gamma}^{1 / 2}\left(\{q\}-q_{h}^{\Gamma}\right)\right\|_{L^{2}(F)}^{2} .
\end{aligned}
$$

It is easy to show that $\|\cdot\|_{D G}$ is a norm if $\sigma_{F}>0$ for all $F \in \mathcal{F}_{h} \backslash \Gamma_{h}$ and that $\|\cdot\|_{\mathcal{E}_{h}}$ is a norm if $\alpha_{\Gamma} \geq 0$ (that is, $\xi>1 / 2$ ).

Further, we give the following definition. 
Definition 5.3. A covering $\mathcal{T}_{\#}=\left\{T_{E}\right\}$ related to the polytopic mesh $\mathcal{T}_{h}$ is a set of shape-regular d-dimensional simplices $T_{E}$ such that for each $E \in \mathcal{T}_{h}$, there exists a $T_{E} \in \mathcal{T}_{\#}$ such that $E \subsetneq T_{E}$.

Finally, for $i=1,2$, we denote by $\mathscr{E}_{i}$ the classical continuous extension operator (cf. [46]), $\mathscr{E}_{i}: H^{s}\left(\Omega_{i}\right) \rightarrow H^{s}\left(\mathbb{R}^{d}\right)$ for $s \in \mathbb{N}_{0}$, and make the following regularity assumptions for the exact solution $\left(p, p_{\Gamma}\right)$ of problem (9).

Assumption 5.4. We assume that the exact solution $\left(\left(p_{1}, p_{2}\right), p_{\Gamma}\right)$ is such that the following hold:

A1. for every $E \in \mathcal{T}_{h}$, if $E \subset \Omega_{i}$, it holds $\left.\mathscr{E}_{i} p_{i}\right|_{T_{E}} \in H^{r_{E}}\left(T_{E}\right)$, with $r_{E} \geq 1+d / 2$ and $T_{E} \in \mathcal{T}_{\#}$ with $E \subset T_{E}$. Moreover, we assume that the normal components of the exact fluxes $\boldsymbol{\nu} \nabla p$ are continuous across internal mesh interfaces, that is, $\llbracket \nu \nabla p \rrbracket=0$ on $\mathcal{F}_{h}^{I}$.

A2. $p_{\Gamma} \in H^{r}(\Gamma)$, with $r \geq 1$.

We can then state the following error estimate.

TheOREM 5.5. Let $\mathcal{T}_{\#}=\left\{T_{E}\right\}$ denote the covering related to $\mathcal{T}_{h}$ consisting of shape-regular simplexes as in Definition 5.3, satisfying Assumption 7.6 (see section 7 below). Let $\left(p, p_{\Gamma}\right)$ be the solution of problem $(9)$ and $\left(p_{h}, p_{h}^{\Gamma}\right) \in V_{h}^{b} \times V_{h}^{\Gamma}$ be its approximation obtained with the method (13) with the penalization parameter given by (17) and $\sigma_{0}$ sufficiently large. Moreover, suppose that the exact solution $\left(p, p_{\Gamma}\right)$ satisfies the regularity Assumption 5.4. Then the following error bound holds:

$$
\begin{aligned}
\left\|\left(p, p_{\Gamma}\right)-\left(p_{h}, p_{h}^{\Gamma}\right)\right\|_{\mathcal{E}_{h}}^{2} \lesssim & \sum_{E \in \mathcal{T}_{h}} \frac{h_{E}^{2\left(s_{E}-1\right)}}{k_{E}^{2\left(r_{E}-1\right)}} G_{E}^{b}\left(h_{E}, k_{E}, \overline{\boldsymbol{\nu}}_{E}\right)\|\mathscr{E} p\|_{H^{r_{E}\left(T_{E}\right)}}^{2} \\
& +\sum_{F \in \Gamma_{h}} \frac{h_{F}^{2 k}}{k^{2(r-1)}}\left|p_{\Gamma}\right|_{H^{r}(F)}^{2},
\end{aligned}
$$

where the $\mathscr{E} p$ is to be interpreted as $\mathscr{E}_{1} p_{1}$ when $E \subset \Omega_{1}$ and as $\mathscr{E}_{2} p_{2}$ when $E \subset \Omega_{2}$. Moreover, $s_{E}=\min \left(k_{E}+1, r_{E}\right)$ and

$$
\begin{aligned}
G_{E}^{b}\left(h_{E}, k_{E}, \overline{\boldsymbol{\nu}}_{E}\right)= & \overline{\boldsymbol{\nu}}_{E}+h_{E} k_{E}^{-1} \max _{F \subset \partial E \backslash \Gamma} \sigma_{F}+\left(\alpha_{\Gamma}+\beta_{\Gamma}\right) h_{E} k_{E}^{-1} \\
& +\overline{\boldsymbol{\nu}}_{E} h_{E}^{-1} k_{E} \max _{F \subset \partial E \backslash \Gamma} \sigma_{F}^{-1}+\overline{\boldsymbol{\nu}}_{E} h_{E}^{-1} k_{E}^{2} \max _{F \subset \partial E \backslash \Gamma} \sigma_{F}^{-1} .
\end{aligned}
$$

Proof. See section 7.

Remark 5.6. If we consider a DG approximation for the problem in the fracture as in (16), using similar techniques, we can prove that the error satisfies the following bounds:

$$
\begin{aligned}
\left\|\left(p, p_{\Gamma}\right)-\left(p_{h}, p_{h}^{\Gamma}\right)\right\|_{\mathcal{E}_{h}}^{2} \lesssim & \sum_{E \in \mathcal{T}_{h}} \frac{h_{E}^{2\left(s_{E}-1\right)}}{k_{E}^{2\left(r_{E}-1\right)}} G_{E}^{b}\left(h_{E}, k_{E}, \overline{\boldsymbol{\nu}}_{E}\right)\|\mathscr{E} p\|_{H^{r_{E}}\left(T_{E}\right)}^{2\left(s_{F}-1\right)} G_{F}^{\Gamma}\left(h_{F}, k_{F}, \overline{\boldsymbol{\nu}}_{F}^{\tau}\right)\left\|\mathscr{E} p_{\Gamma}\right\|_{H^{r_{F}}\left(T_{F}\right)}^{2}, \\
& +\sum_{F \in \Gamma_{h}} \frac{h_{F}^{2\left(r_{F}-1\right)}}{k_{F}^{2\left(r^{2}\right.}}
\end{aligned}
$$

where

$$
\begin{aligned}
G_{F}^{\Gamma}\left(h_{F}, k_{F}, \overline{\boldsymbol{\nu}}_{F}^{\tau}\right)= & \overline{\boldsymbol{\nu}}_{F}^{\tau} \ell_{\Gamma}+h_{F} k_{F}^{-1} \max _{e \subseteq \partial F} \sigma_{e}+\alpha_{\Gamma} h_{F}^{2} k_{F}^{-2} \\
& +\left(\overline{\boldsymbol{\nu}}_{F}^{\tau} \ell_{\Gamma}\right)^{2} h_{F}^{-1} k_{F} \max _{e \subseteq \partial F} \sigma_{e}^{-1}+\left(\overline{\boldsymbol{\nu}}_{F}^{\tau} \ell_{\Gamma}\right)^{2} h_{F}^{-1} k_{F}^{2} \max _{e \subseteq \partial F} \sigma_{e}^{-1} .
\end{aligned}
$$

Copyright (c) by SIAM. Unauthorized reproduction of this article is prohibited. 
6. Numerical results. In this section we present some two-dimensional numerical experiments to confirm the validity of the a priori error estimates that we have derived for our method. The test cases have been chosen intentionally with increasing complexity: We start with some academic numerical tests that aim at validating the convergence properties of the method, we continue the section with a physical experiment that investigates the effect of large and low permeability in the fracture to the bulk flow, and we end the section with numerical experiments aiming at assessing the capability of our method to deal with more complex geometrical configurations, namely, partially immersed fractures and network of intersecting fractures.

The numerical results have been obtained in MATLAB. For the generation of polygonal meshes conforming to the fractures, we have suitably modified the MATLAB code PolyMesher developed by G.H. Paulino and collaborators [47].

6.1. Example 1. In this first test case we take $\Omega=(0,1)^{2}$, and choose as exact solutions in the bulk and in the fracture $\Gamma=\{(x, y) \in \Omega: x+y=1\}$ as

$$
p=\left\{\begin{array}{ll}
e^{x+y} & \text { in } \Omega_{1}, \\
\frac{e^{x+y}}{2}+\left(\frac{1}{2}+\frac{3 \eta_{\Gamma}}{\sqrt{2}}\right) e & \text { in } \Omega_{2},
\end{array} \quad p_{\Gamma}=e\left(1+\sqrt{2} \eta_{\Gamma}\right) .\right.
$$

It is easy to prove that $p$ and $p_{\Gamma}$ satisfy the coupling conditions (5a)-(5b) with $\xi=1$ and $\boldsymbol{\nu}=\mathbf{I}$. Finally, we need to adjust the source terms for the bulk and fracture problems accordingly:

$$
f=\left\{\begin{array}{ll}
-2 e^{x+y} & \text { in } \Omega_{1}, \\
-e^{x+y} & \text { in } \Omega_{2}
\end{array} \quad f_{\Gamma}=\frac{e}{\sqrt{2}} .\right.
$$

Notice that on the fracture, the source term satisfies $f_{\Gamma}=-\nabla_{\tau} \cdot\left(\boldsymbol{\nu}_{\Gamma}^{\tau} \ell_{\Gamma} \nabla_{\tau} p_{\Gamma}\right)+\llbracket \nu \nabla p \rrbracket$, and, since $p_{\Gamma}$ is constant, it holds that $f_{\Gamma}=\llbracket \nu \nabla p \rrbracket$.

Figure 2 shows the computed errors $\left\|p-p_{h}\right\|_{D G}$ for the bulk problem and the corresponding computed errors $\left\|p^{\Gamma}-p_{h}^{\Gamma}\right\|_{\Gamma}$ in the fracture. The results have been obtained taking the polynomial degree $k=1$ for both the bulk and the fracture problems. As predicted from our theoretical error bounds, a convergence of order 1

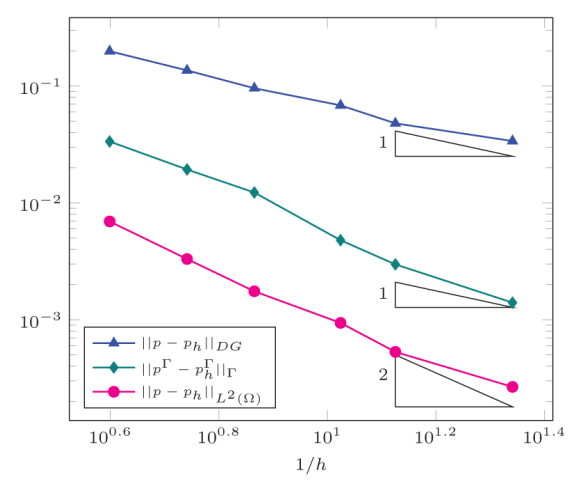

(a) Bulk and fracture $k=1$

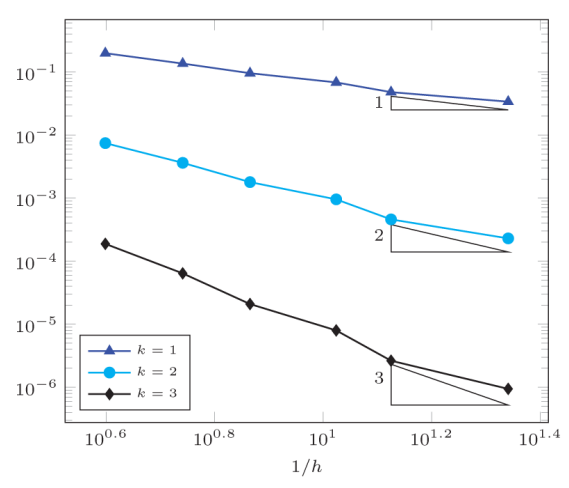

(b) Bulk $k=1,2,3$

FIG. 2. Example 1. (a) Computed errors in the bulk and in the fracture as a function of the inverse of the mesh size (log-log scale) with polynomial degree $k=1$. (b) Computed errors $\left\|p-p_{h}\right\|_{D G}$ in the bulk for polynomial degrees $k=1,2,3$. 
is clearly observed for both $\left\|p-p_{h}\right\|_{D G}$ and $\left\|p^{\Gamma}-p_{h}^{\Gamma}\right\|_{\Gamma}$. Moreover, from Figure 2 one can clearly see that also in this test case, one order of convergence is gained if we compute the error $\left\|p-p_{h}\right\|_{L^{2}(\Omega)}$. In Figure 2 we plot the computed errors in the bulk $\left\|p-p_{h}\right\|_{D G}$ for polynomial degrees $k_{E}=k=1,2,3$. They are in agreement with the expected convergence rates of $\mathcal{O}\left(h^{k}\right)$.

6.2. Example 2. In this second example we consider the circular fracture $\Gamma=$ $\left\{(x, y) \in \Omega: x^{2}+y^{2}=R\right\}$ with $R=0.7$ included in the domain $\Omega=(0,1)^{2}$. We choose the exact solutions in the bulk and in the fracture as

$$
p=\left\{\begin{array}{ll}
\frac{x^{2}+y^{2}}{R^{2}} & \text { in } \Omega_{1}, \\
\frac{x^{2}+y^{2}}{2 R^{2}}+\frac{3}{R} \eta_{\Gamma}+\frac{1}{2} & \text { in } \Omega_{2},
\end{array} p_{\Gamma}=1+\frac{7}{4} \frac{\eta_{\Gamma}}{R},\right.
$$

so that they satisfy the coupling conditions (5a)-(5b) with $\xi=\frac{3}{4}$ and $\boldsymbol{\nu}=\mathbf{I}$. The source term is chosen as

$$
f=\left\{\begin{array}{ll}
-\frac{4}{R^{2}} & \text { in } \Omega_{1}, \\
-\frac{2}{R^{2}} & \text { in } \Omega_{2}
\end{array} \quad f_{\Gamma}=\frac{1}{R}\right.
$$

Figure 3 shows an example of mesh grid employed in this set of experiments. One can see that here the fracture is approximated by a polygonal line.

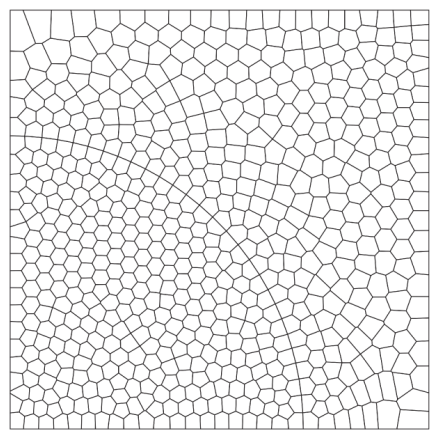

(a) Mesh grid

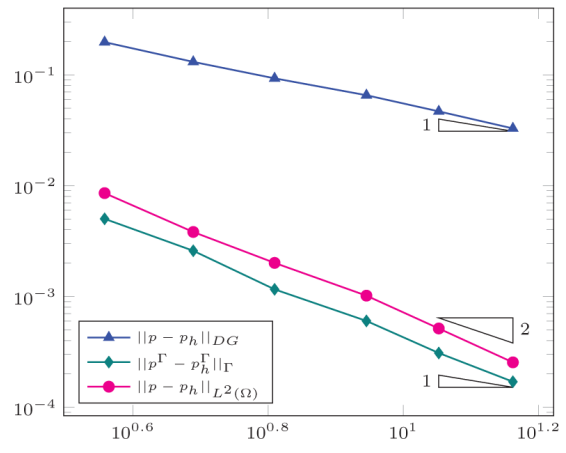

(b) Errors

FIG. 3. Example 2. (a) Example of the polygonal mesh grid with circular fracture. (b) Computed errors as a function of inverse of the mesh size (log-log scale) with polynomial degree $k=1$.

In Figure 3 we report the computed errors $\left\|p-p_{h}\right\|_{D G}$ and $\left\|p^{\Gamma}-p_{h}^{\Gamma}\right\|_{\Gamma}$ as a function of $1 / h$ for $k_{E}=k=1$ (we disregard the variational crime coming from the polygonal approximation of the circular fracture). The numerical experiments validate the theoretical estimates, as a linear decay of the error is clearly observed.

6.3. Example 3. We consider the domain $\Omega=(0,1)^{2}$ and the fracture $\Gamma=$ $\{(x, y) \in \Omega: \quad x=0.5\}$. Following [27], we choose the exact solutions in the bulk and in the fracture as

$$
p=\left\{\begin{array}{ll}
\sin (4 x) \cos (\pi y) & \text { if } x<0.5, \\
\cos (4 x) \cos (\pi y) & \text { if } x>0.5,
\end{array} \quad p_{\Gamma}=\frac{3}{4}[\cos (2)+\sin (2)] \cos (\pi y),\right.
$$

so that they satisfy the coupling conditions $(5 \mathrm{a})-(5 \mathrm{~b})$ with $\xi=\frac{3}{4}$ and $\boldsymbol{\nu}=\mathbf{I}$. We also choose the fracture thickness to be equal to $\ell_{\Gamma}=0.25$ and the tangential and normal components of the permeability tensor in the fracture to be $\boldsymbol{\nu}_{\Gamma}^{\tau}=1$ and $\nu_{\Gamma}^{n}=1$, 


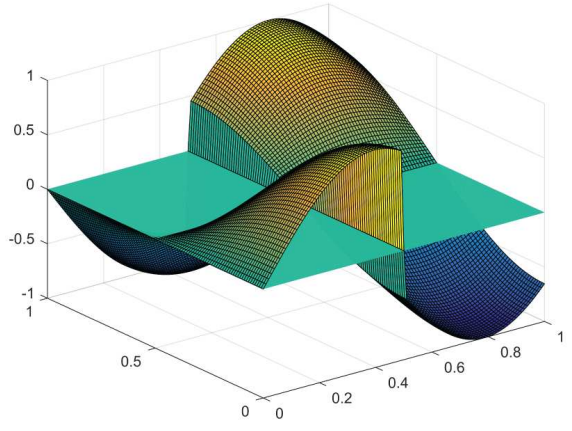

(a) Exact solution

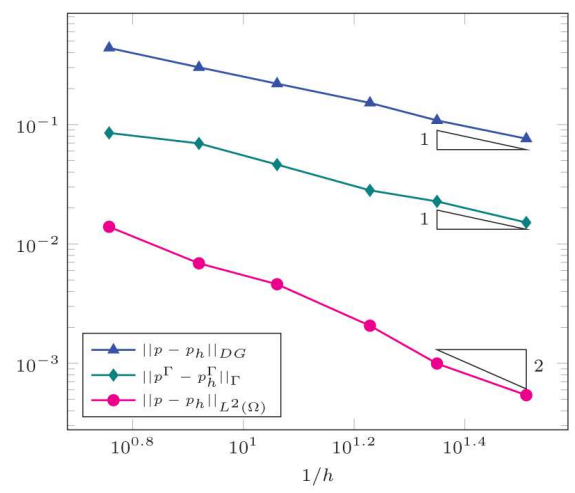

(b) Errors

FIG. 4. Example 3. Exact solution in the bulk with the plane $x=y=0$ (left) and computed errors as a function of inverse of the mesh size (log-log scale) with polynomial degree $k=1$ (right).

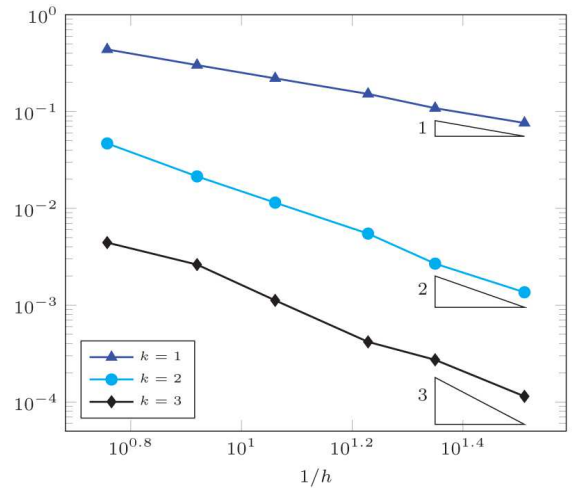

(a)

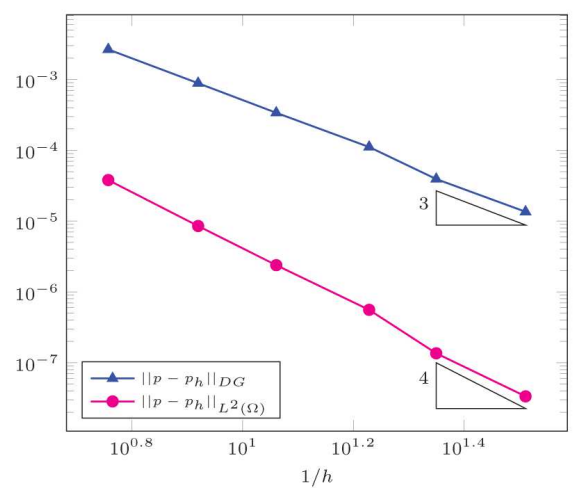

(b)

FIG. 5. Example 3. (a) Computed errors $\left\|p-p_{h}\right\|_{D G}$ in the bulk as a function of the inverse of the mesh size (log-log scale) for polynomial degrees $k=1,2,3$. (b) Computed errors in the bulk with polynomial degree $k=3$ and known fracture pressure.

respectively. We impose Dirichlet boundary conditions on the whole $\partial \Omega$ and also on $\partial \Gamma$. Finally, the source terms are chosen accordingly as

$f=\left\{\begin{array}{ll}\sin (4 x) \cos (\pi y)\left(16+\pi^{2}\right) & \text { if } x<0.5, \\ \cos (4 x) \cos (\pi y)\left(16+\pi^{2}\right) & \text { if } x>0.5,\end{array} f_{\Gamma}=\ell_{\Gamma} \cos (\pi y)[\cos (2)+\sin (2)]\left(4+\frac{3}{16} \pi^{2}\right)\right.$.

The exact solution in the bulk is shown in Figure 4. In Figure 4 we show the computed errors in the bulk and in the fracture for polynomial degree equal to 1 for both the bulk and the fracture problems. In Figure 5 we report the errors $\left\|p-p_{h}\right\|_{D G}$ obtained with polynomial degrees $k_{E}=1,2,3$ in the bulk and $k=1$ in the fracture. We observe that for $k_{E}=3$ the convergence rate is suboptimal. This is due to the fact that the polynomial degree in the fracture is not accurate enough. In fact, if we assume to know the exact solution $p_{\Gamma}$ in the fracture and we solve the problem in the bulk, we recover the expected rates, as shown in Figure 5. This behavior did not appear in the previous test cases, where the solution in the fracture was chosen to be constant. 
6.4. Quarter five-spot problem. The quarter five-spot problem is often used to validate numerical schemes for the approximation of Darcy's flow; see, for example, $[27,39]$. A five-spot is a standard technique used in petroleum engineering for oil recovery, where four injection wells are located at the corner of a square and one production well is located in its center. Fluid (typically water, steam, or gas) is injected simultaneously through the four injection wells causing the displacement of the oil toward the production well in the center. Since the problem is symmetric, we can consider only a quarter of this injection pattern, represented by the domain $\Omega=(0,1)^{2}$. The single injection well will be then located in $(0,0)$ and the production well in $(1,1)$. Their presence is included in the model via the source term

$$
f(x)=10.1\left[\tan \left(200\left(0.2-\sqrt{x^{2}+y^{2}}\right)\right)-\tanh \left(200\left(0.2-\sqrt{(x-1)^{2}+(y-1)^{2}}\right)\right)\right] .
$$

Moreover, we enforce homogeneous Neumann and Dirichlet boundary conditions, respectively, on $\partial \Omega_{N}=\{x=0$ or $y=0\}$ and $\partial \Omega_{D}=\{x=1$ or $y=1\}$. We also assume that the domain is cut by the fracture of equation $\Gamma=\{(x, y) \in \Omega: \quad x+y=1\}$ with thickness $\ell_{\Gamma}=0.005$, and we let $f_{\Gamma}=0$. Finally, we impose homogeneous Dirichlet boundary conditions on $\partial \Gamma$. The domain configuration is reported in Figure 6(a) for clarity. We aim, in particular, at investigating the effect of large and small permeability in the fracture to the overall flow. We perform two numerical experiments:

1. Permeable fracture: We choose $\nu_{\Gamma}^{n}=1$ and $\boldsymbol{\nu}_{\Gamma}^{\tau}=100$.

2. Impermeable fracture: We choose $\nu_{\Gamma}^{n}=10^{-2}$ and $\boldsymbol{\nu}_{\Gamma}^{\tau}=1$.

In both cases we let the bulk permeability tensor $\boldsymbol{\nu}=\mathbf{I}$, and we solve the problem choosing a polygonal mesh with $h=7.5 \cdot 10^{-2}$ and the polynomial degree $k_{E}=2$ in the bulk and $k=1$ in the fracture.

The bulk pressures obtained are shown in Figure 7. As expected, in both cases the bulk pressure has a peak in correspondence of injection well, and it decreases going toward the production well. In the permeable case, the decrease is continuous, while in the impermeable case we can observe a clear jump of the pressure across the fracture. This behavior is better captured in Figure 6(b), where we have plotted the trend of the pressure along the line $x=y$ in both cases. Our qualitative results are in agreement with those obtained in [27].

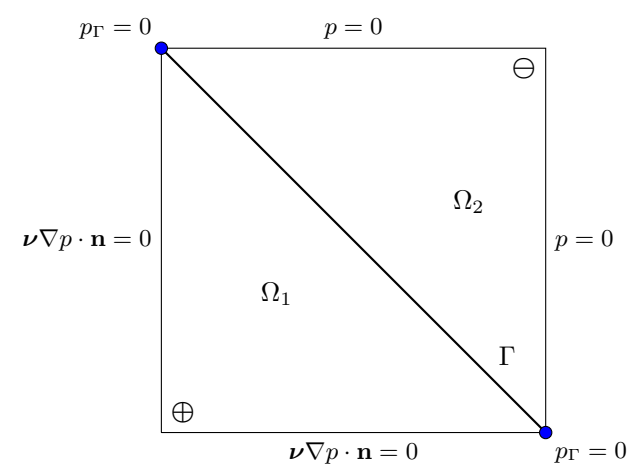

(a)

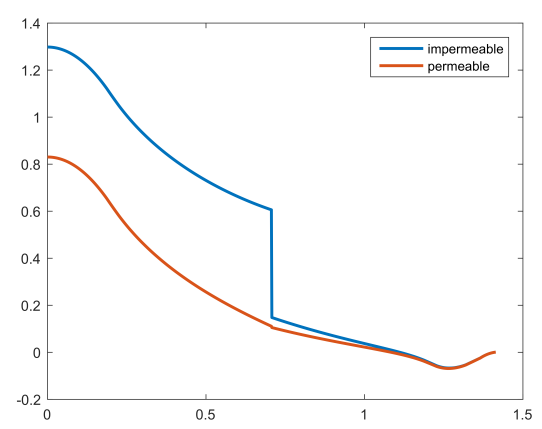

(b)

FIG. 6. Quarter five-spot. The subdomains $\Omega_{1}$ and $\Omega_{2}$ separated by the fracture $\Gamma$ and boundary conditions (left) and pressure in the bulk along the line $x=y$ (right). 


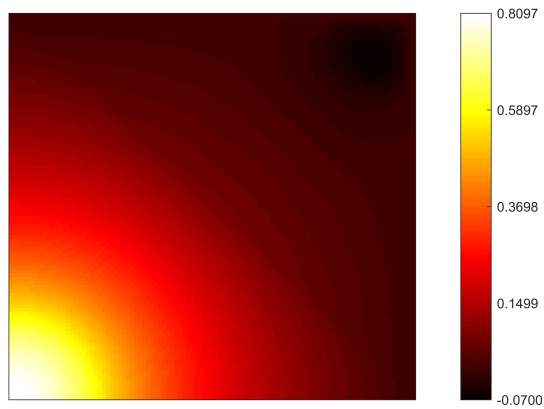

(a) Permeable fracture

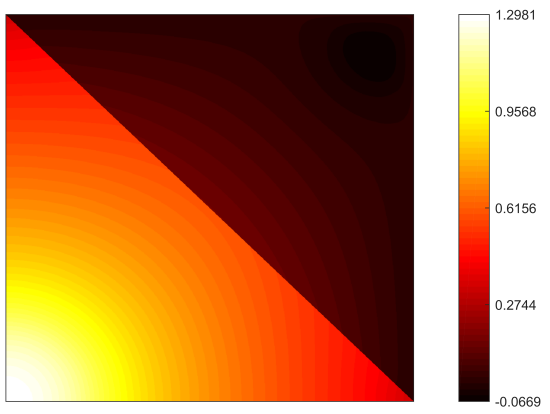

(b) Impermeable fracture

FIG. 7. Quarter five-spot. Pressure in the bulk for the quarter-five spot problem with permeable (left) and impermeable (right) fracture.

6.5. Immersed fractures. We now investigate the capability of our discretization method to deal with immersed fractures. We take as a reference [3], where the model developed in [42] has been extended to fully immersed fractures. In particular, our set of equations (7) needs to be supplemented with a condition on the boundary $\partial \Gamma$ immersed in the porous medium. Following [3], we will assume that the mass transfer across the immersed tip can be neglected, imposing the Neumann boundary condition $\boldsymbol{\nu}_{\Gamma}^{\tau} \nabla_{\tau} p_{\Gamma} \cdot \boldsymbol{\tau}=0$ on $\partial \Gamma$. On the fracture tip intersecting the bulk boundary, i.e., $\partial \Gamma \cap \partial \Omega$, we impose boundary conditions coincident with those imposed on $\partial \Omega$.

We perform two sets of numerical experiments that were already proposed in [3]. The aim is that of investigating the flow depending on the physical properties of the fractures (permeable, impermeable), first in the case of a single fracture and then in the more complex situation of a network of partially immersed fractures. Our results are in perfect agreement with those obtained in [3], thus showing that our method can be easily extended to the treatment of more complex and realistic situations.

For all the experiments we take as computational domain $\Omega=(0,1)^{2}$, and we assume that the bulk permeability tensor is isotropic, i.e., $\boldsymbol{\nu}=\mathrm{Id}$. Moreover, we take the forcing terms $f=f_{\Gamma}=0$, so that the flow is only generated by boundary conditions. Finally, we choose the parameter $\xi=0.55$. Our results have been obtained with cartesian grids with approximately the same number of elements as those employed in [3]. Note that the grids are aligned with the fractures so that the immersed tips coincide with one of the mesh vertices.

6.5.1. Single partially immersed fracture. In the first experiment we study the case when the porous medium is cut by the fracture $\Gamma=\left\{(x, y) \in[0,1]^{2}: x=\right.$ $0.5, y \geq 0.5\}$ that is partially immersed in the domain and has constant aperture $\ell_{\Gamma}=0.01$. We consider two different configurations where we vary the boundary conditions and the permeability of the fracture:

1. Permeable fracture: We choose $\nu_{\Gamma}^{n}=100$ and $\boldsymbol{\nu}_{\Gamma}^{\tau}=10^{6}$ and impose Dirichlet boundary condition on the whole $\partial \Omega$ as described in Figure 8(a).

2. Impermeable fracture: We choose $\nu_{\Gamma}^{n}=\boldsymbol{\nu}_{\Gamma}^{\tau}=10^{-7}$ and impose mixed boundary conditions on $\partial \Omega$ as in Figure 8(b).

The results obtained with a mesh of 16,128 elements are shown in Figure 9. In both cases, on the left part of the figure, we show the pressure field in the bulk (where the intensity of the color increases with the increasing of the pressure) together with 


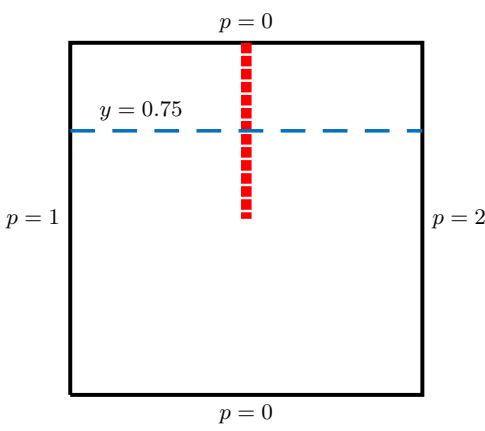

(a) Permeable fracture

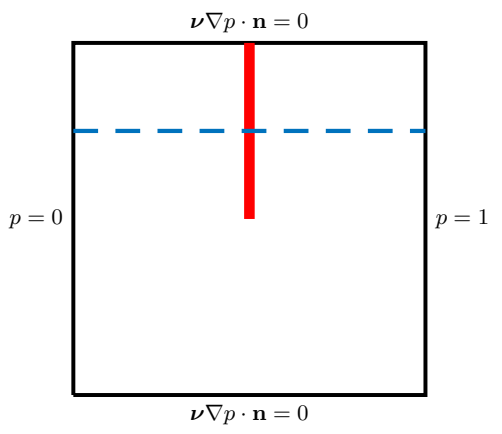

(b) Impermeable fracture

FIG. 8. Single immersed fracture. Configurations and boundary conditions for the permeable test case (left) with $\boldsymbol{\nu}_{\Gamma}^{\tau}=10^{6}, \nu_{\Gamma}^{n}=10^{2}$ and the impermeable test case (right) with $\boldsymbol{\nu}_{\Gamma}^{\tau}=\nu_{\Gamma}^{n}=10^{-7}$.

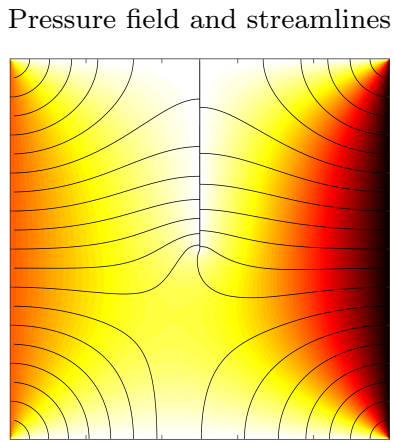

Pressure along $y=0.75$
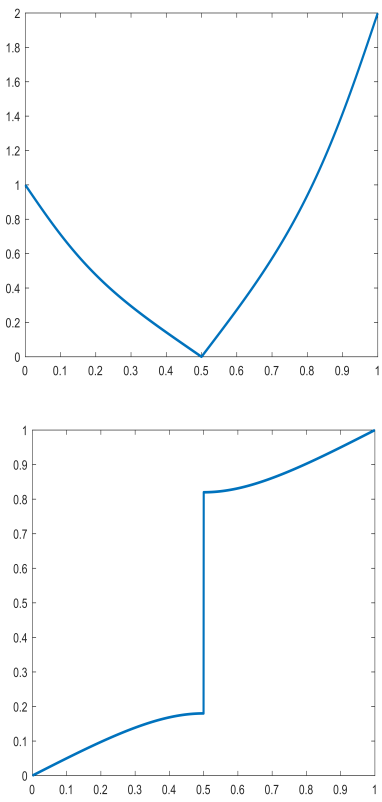

Pressure in the fracture
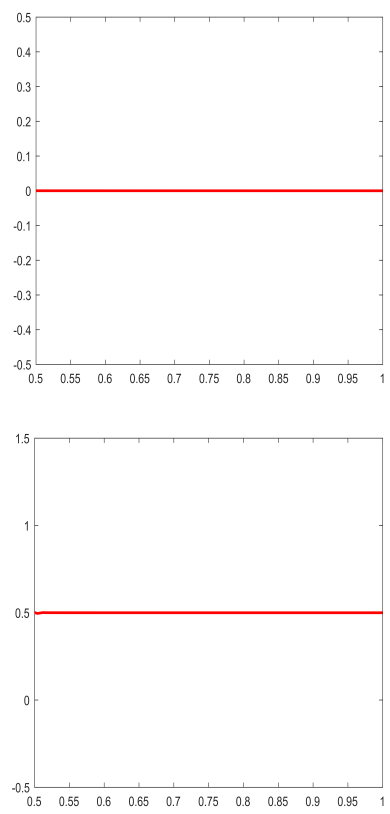

FIG. 9. Single immersed fracture. Permeable case (top) and impermeable case (bottom).

the streamlines of Darcy's velocity. In the middle, we report the behavior of the bulk pressure along the line $y=0.75$. Finally, on the right we plot the pressure field in the fracture. As expected, in the impermeable case we can observe a clear jump of the bulk pressure across the fracture that is not present in the permeable case. The results presented in Figure 9 are in agreement with those of [3].

6.5.2. Network of partially immersed fractures. In the second experiment, we consider a network of four partially immersed fractures of aperture $\ell_{\Gamma}=0.01$, namely, $\Gamma_{1}=\left\{(x, y) \in[0,1]^{2}: x \geq 0.3, y=0.2\right\}, \Gamma_{2}=\left\{(x, y) \in[0,1]^{2}: x \leq 0.7, y=0.4\right\}$, $\Gamma_{3}=\left\{(x, y) \in[0,1]^{2}: x \geq 0.3, y=0.6\right\}$, and $\Gamma_{4}=\left\{(x, y) \in[0,1]^{2}: x \leq 0.7, y=0.8\right\}$. 


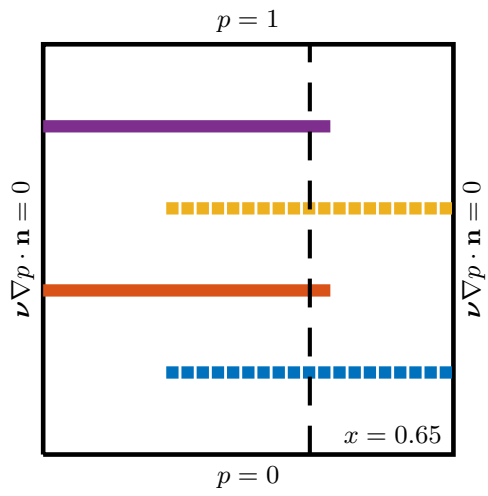

(a) Configuration 1: $\boldsymbol{\nu}_{\Gamma}^{\tau}=100$ on $\Gamma_{1}, \Gamma_{3}$

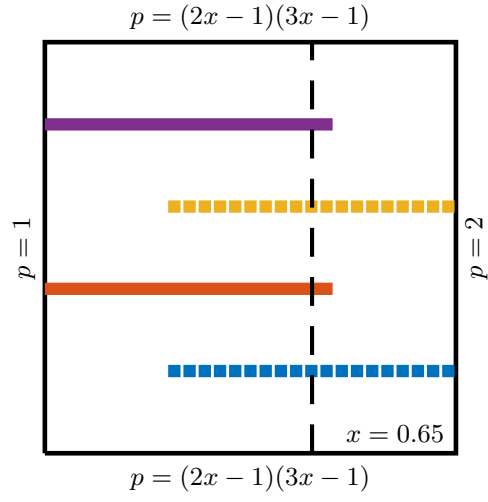

(b) Configuration 2: $\boldsymbol{\nu}_{\Gamma}^{\tau}=1$ on $\Gamma_{1}, \Gamma_{3}$

FIG. 10. Network of immersed fractures. Configurations and boundary condition for the two test cases.

The fractures $\Gamma_{2}$ and $\Gamma_{4}$ are impermeable $\left(\boldsymbol{\nu}_{\Gamma}^{\tau}=\nu_{\Gamma}^{n}=10^{-2}\right)$, while $\Gamma_{1}$ and $\Gamma_{3}$ are partially permeable $\left(\nu_{\Gamma}^{n}=10^{-2}, \boldsymbol{\nu}_{\Gamma}^{\tau} \in\{100,1\}\right)$. We consider two different configurations, varying the value of the permeability $\boldsymbol{\nu}_{\Gamma}^{\tau}$ on the partially permeable fractures $\Gamma_{1}$ and $\Gamma_{3}$ and the boundary conditions as illustrated in Figure 10.

In Figure 11, we show the results obtained for the two test cases with a mesh of 26,051 elements. In particular, we report the pressure field in the bulk with the

Pressure field and streamlines
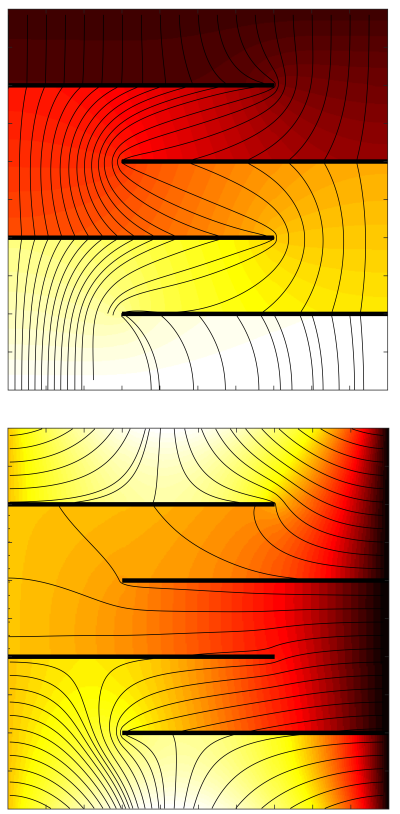

Pressure along $y=0.75$
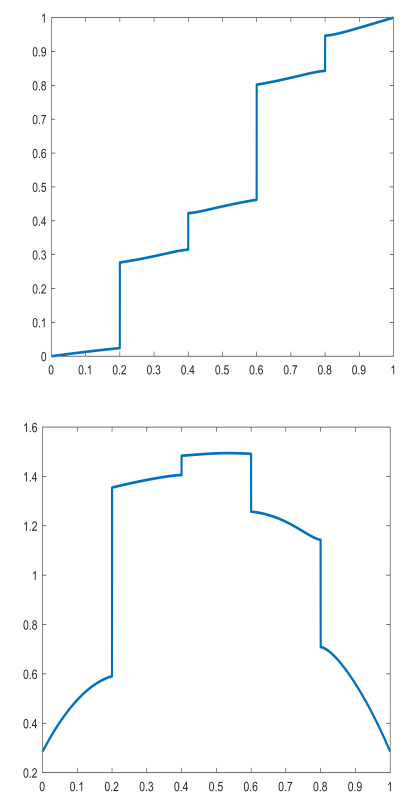

Pressure in the fracture
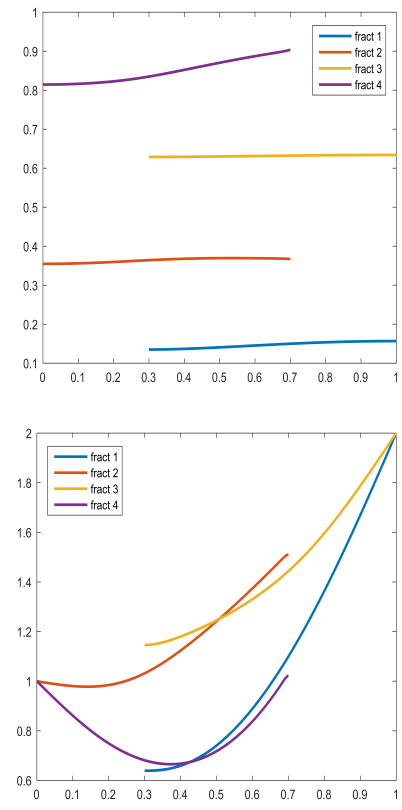

FIG. 11. Network of immersed fractures. First configuration (top) and second configuration (bottom). 

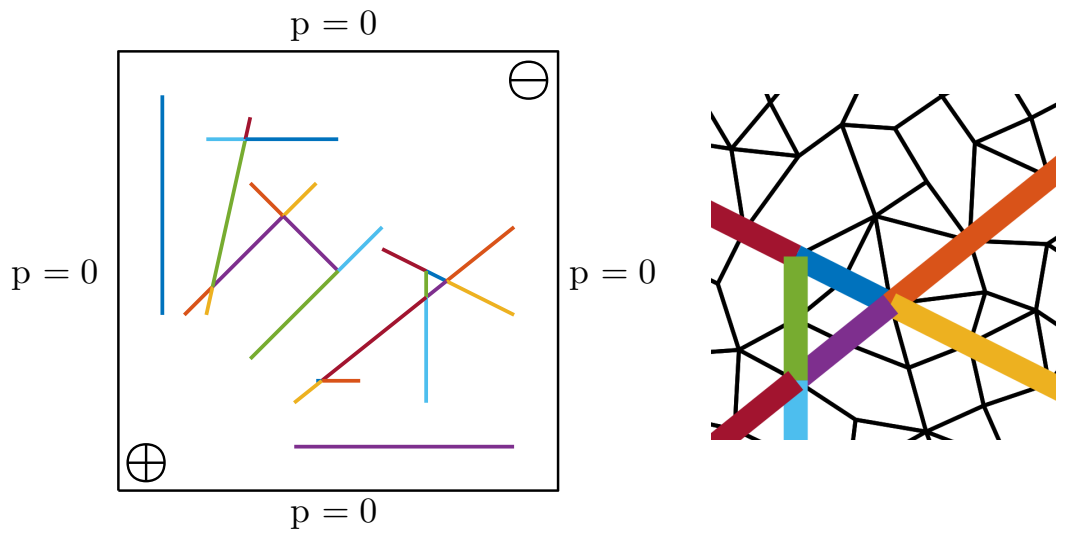

FIG. 12. Network of intersecting fractures. Computational domanin and zoomed detail of the polygonal mesh employed for the computations.

streamlines of the velocity (left), the value of the bulk pressure along the line $x=0.65$ (middle), and the pressure field inside the four fractures (right). Again, we can see a perfect agreement between our results and those obtained in [3].

6.6. Network of intersecting fractures. The aim of this section is to investigate the capability of our method to deal with networks of intersecting fractures. To do so, we need to complete the mathematical model (7) with some conditions at the intersection points. One possible choice is to impose pressure continuity and flux conservation, as in $[45,18]$. At the immersed tips we impose the no-flux condition $\boldsymbol{\nu}_{\Gamma}^{\tau} \nabla_{\tau} p_{\Gamma} \cdot \boldsymbol{\tau}=0$ as above.

We reproduce the numerical experiments performed with mimetic finite differences in [6]. We consider the domain $\Omega=(0,1)^{2}$ containing 10 intersecting fractures. The geometry of the problem is shown in Figure 12, where the fractures are highlighted with colored lines. We remark that for the computations we have employed a mesh made of general polygonal elements, as shown in the zoomed detail reported in Figure 12.

We impose homogeneous Dirichlet boundary conditions on the whole $\partial \Omega$ and define the source term as

$$
f(x, y)= \begin{cases}10 & \text { if }(x-0.1)^{2}+(y-0.1)^{2} \leq 0.04 \\ -10 & \text { if }(x-0.9)^{2}+(y-0.9)^{2} \leq 0.04\end{cases}
$$

so that we have a source in the lower left corner of the domain and a sink in its top right corner. The porous medium in the bulk is isotropic and homogeneous, i.e., $\boldsymbol{\nu}=$ Id. The fractures are isotropic, i.e., $\boldsymbol{\nu}_{\Gamma}^{\tau}=\nu_{\Gamma}^{n}$, with constant thickness $\ell_{\Gamma}=0.01$. We consider three test cases:

1. No fractures are present in the porous medium.

2. Permeable network: All the fractures have high-permeability properties, taking $\boldsymbol{\nu}_{\Gamma}^{\tau}=\nu_{\Gamma}^{n}=1000$.

3. Impermeable network: All the fractures have blocking properties, taking $\boldsymbol{\nu}_{\Gamma}^{\tau}=\nu_{\Gamma}^{n}=0.001$

In all the test cases, we take $\xi=0.75$. The discrete pressures for the problem in the bulk are reported in Figure 13. For the computation of the discrete solution we have employed a DG scheme with polynomial degree $k=2$ for the bulk problem and a DG 


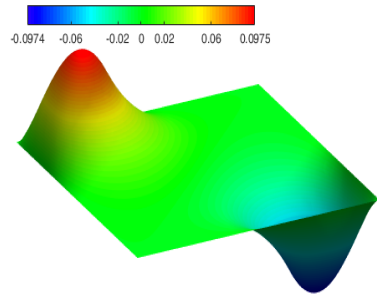

(a) No fractures

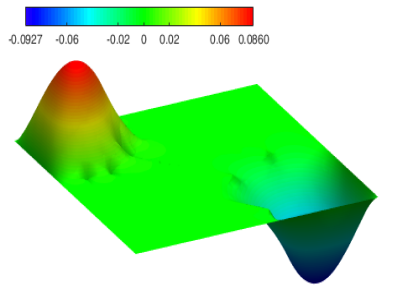

(b) Permeable

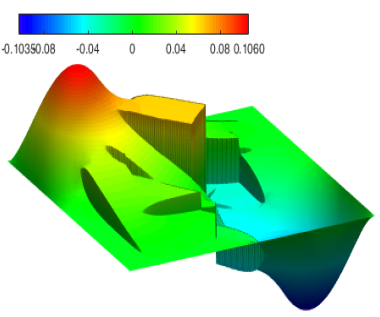

(c) Impermeable

Fig. 13. Network of intersecting fractures. Discrete pressure in the bulk for the three test cases: no fractures (left), permeable network $\nu_{\Gamma}^{\tau}=\nu_{\Gamma}^{n}=1000$ (middle), and impermeable network $\nu_{\Gamma}^{\tau}=\nu_{\Gamma}^{n}=0.001$ (right).

scheme on each fracture problem with polynomial degree $k_{\Gamma}=2$. We observe that, in all the cases, the results are consistent with those obtained in [45] with mimetic finite differences. In the permeable case (see Figure 13(b)) the pressure is almost continuous across the fractures, as expected. In agreement with [45], the maximum and minimum values reached by the pressure are slightly lower than those of the nonfractured case; see Figure 13(a). In the impermeable case, we observe clear jumps of the bulk pressure across the fractures; see Figure 13(c). Once again, our results are in good agreement with those obtained in [45].

7. Theoretical analysis. This section contains all the technical details needed for the proof of the well-posedness of the descrete formulation (cf. Theorem 5.2) and of the error estimates (cf. Theorem 5.5).

7.1. Stability analysis. We first consider well-posedness. Since our formulation employs general polytopes, we will first introduce some technical results to treat such kind of discretizations; cf. [25, 23, 4, 22, 9].

7.1.1. Trace inverse estimates. Trace inverse estimates bound the norm of a polynomial on an element's face/edge by the norm on the element itself. They are at the base of the stability and error analysis of DG methods. The use of grids made of general polytopic elements presents challenges on a number of points. Indeed, in contrast to the case when standard-shaped elements are employed, shape-regular polytopes may admit an arbitrary number of faces/edges, and the measure of the faces/edges may potentially be much smaller than the measure of the element itself. In order to obtain an inverse estimate valid on polygons/polyhedra which is sharp with respect to facet degeneration and holds true even when the number of faces/edges is unbounded, taking as a reference [22], we make the following assumption on the mesh.

Assumption $7.1([22])$. For any $E \in \mathcal{T}_{h}$, there exists a set of nonoverlapping (not necessarily shape-regular) d-dimensional simplices $\left\{S_{E}^{i}\right\}_{i=1}^{n_{E}}$ contained in $E$ such that $\bar{F}=\partial \bar{E} \cap \overline{S_{E}^{i}}$ for any face $F \subseteq \partial E$ and

$$
h_{E} \lesssim \frac{d\left|S_{E}^{i}\right|}{|F|}, \quad i=1, \ldots, n_{E}
$$

Here, the hidden constant is independent of the discretization parameters, the number of faces of the element $n_{E}$, and the face measure. 
Note that this assumption does not give any restriction on the number of faces per element or on the measure of the faces. We also underline that the union of simplices $S_{E}^{i}$ does not have to cover, in general, the whole element $E$, that is,

$$
\cup_{i=1}^{n_{E}} \bar{S}_{E}^{i} \subseteq \bar{E}
$$

In the following, for simplicity and clarity we shall write $S_{E}^{F}$ instead of $S_{E}^{i}$.

First, we recall a classical $h p$-version inverse estimate valid for generic simplices [48].

Lemma 7.2. Let $S \subset \mathbb{R}^{d}$ be a simplex, and let $v \in \mathbb{P}_{k}(S)$. Then, for each $F \subset \partial S$, we have

$$
\|v\|_{L^{2}(F)}^{2} \leq \frac{(k+1)(k+d)}{d} \frac{|F|}{|S|}\|v\|_{L^{2}(S)}^{2} .
$$

The inverse estimate for polytopic elements is then obtained using Assumption 7.1 as in [22, Lemma 4.1] and [9, 24]. The proof is reported here for completeness.

Lemma 7.3. Let $E$ be a polygon/polyhedron satisfying Assumption 7.1, and let $v \in \mathbb{P}_{k_{E}}(E)$. Then we have

$$
\|v\|_{L^{2}(\partial E)}^{2} \lesssim \frac{k_{E}^{2}}{h_{E}}\|v\|_{L^{2}(E)}^{2}
$$

where the hidden constant depends on the dimension d, but it is independent of the discretization parameters and of the number of faces of the element.

Proof. The proof follows immediately if we apply Lemma 7.2 to each simplex $S_{E}^{F} \subset E$ from Assumption 7.1, together with (19). More in detail, we have

$$
\begin{aligned}
\|v\|_{L^{2}(\partial E)}^{2} & =\sum_{F \subset \partial E}\|v\|_{L^{2}(F)}^{2} \lesssim k_{E}^{2} \sum_{F \subset \partial E} \frac{|F|}{\left|S_{E}^{F}\right|}\|v\|_{L^{2}\left(S_{E}^{F}\right)}^{2} \lesssim \frac{k_{E}^{2}}{h_{E}}\|v\|_{L^{2}\left(\bigcup_{F \subset \partial E} S_{E}^{F}\right)}^{2} \\
& \leq \frac{k_{E}^{2}}{h_{E}}\|v\|_{L^{2}(E)}^{2} .
\end{aligned}
$$

Note that the estimate bounds the $L^{2}$-norm of the polynomial on the whole boundary of $E$, not just on one of its edges/faces. This will be of fundamental importance in the analysis.

7.1.2. Well-posedness of the discrete formulation. We can now proceed with the stability analysis of our method. We recall that we are supposing that the permeability tensors $\boldsymbol{\nu}$ and $\boldsymbol{\nu}_{\Gamma}$ are piecewise constant on mesh elements.

Following [25, 23, 4, 22], we base our analysis on the introduction of an appropriate inconsistent formulation for the problem in the bulk. This choice is determined by the necessity of avoiding to make further (unnatural) regularity requirements for the exact solution; cf. section 7.2.1 below. To this end we define the following extension of the forms $\mathcal{A}_{b}^{D G}$ and $\mathcal{L}_{b}^{D G}$ :

Copyright $@$ by SIAM. Unauthorized reproduction of this article is prohibited. 


$$
\begin{aligned}
\tilde{\mathcal{A}}_{b}^{D G}(p, q)= & \sum_{E \in \mathcal{T}_{h}} \int_{E} \nu \nabla p \cdot \nabla q-\sum_{F \in \mathcal{F}_{h} \backslash \Gamma_{h}} \int_{F}\left\{\boldsymbol{\nu} \Pi_{2}(\nabla p)\right\} \cdot \llbracket q \rrbracket \\
& -\sum_{F \in \mathcal{F}_{h} \backslash \Gamma_{h}} \int_{F}\left\{\boldsymbol{\nu} \Pi_{2}(\nabla q)\right\} \cdot \llbracket p \rrbracket+\sum_{F \in \mathcal{F}_{h} \backslash \Gamma_{h}} \sigma_{F} \int_{F} \llbracket p \rrbracket \cdot \llbracket q \rrbracket, \\
\tilde{\mathcal{L}}_{b}^{D G}(q)= & \sum_{E \in \mathcal{T}_{h}} \int_{E} f q+\left[\sum_{F \in \mathcal{F}_{h}^{B}} \int_{F}\left(-\nu \Pi_{2}(\nabla q) \cdot \mathbf{n}_{F}+\sigma_{F} q\right) g\right],
\end{aligned}
$$

where the integral between square brackets vanishes if we consider homogeneous boundary conditions. Here, $\Pi_{2}:\left[L^{2}(\Omega)\right]^{d} \rightarrow\left[V_{h}^{b}\right]^{d}$ denotes the orthogonal $L^{2}$ projection onto the bulk finite element space $\left[V_{h}^{b}\right]^{d}$. It follows that these forms are well defined on the space $V^{b}(h)=V_{h}^{b}+V^{b}$ since the terms $\left\{\boldsymbol{\nu} \Pi_{2}(\nabla q)\right\}$ and $\left\{\boldsymbol{\nu} \Pi_{2}(\nabla p)\right\}$ are traces of elementwise polynomial functions. Moreover, it is clear that

$$
\tilde{\mathcal{A}}_{b}^{D G}\left(p_{h}, q_{h}\right)=\mathcal{A}_{b}^{D G}\left(p_{h}, q_{h}\right) \quad \text { for all } q_{h}, p_{h} \in V_{h}^{b}
$$

and

$$
\tilde{\mathcal{L}}_{b}^{D G}\left(q_{h}\right)=\mathcal{L}_{b}^{D G}\left(q_{h}\right) \text { for all } q_{h} \in V_{h}^{b} .
$$

Thereby, $\tilde{\mathcal{A}}_{b}^{D G}(\cdot, \cdot)$ and $\tilde{\mathcal{L}}_{b}^{D G}(\cdot)$ are extensions of $\mathcal{A}_{b}^{D G}(\cdot, \cdot)$ and $\mathcal{L}_{b}^{D G}(\cdot)$ to $V^{b}(h) \times V^{b}(h)$ and $V^{b}(h)$, respectively. Hence, we may rewrite our discrete problem (13) in the following equivalent form.

Find $\left(p_{h}, p_{h}^{\Gamma}\right) \in V_{h}^{b} \times V_{h}^{\Gamma}$ such that

$$
\tilde{\mathcal{A}}_{h}\left(\left(p_{h}, p_{h}^{\Gamma}\right),\left(q_{h}, q_{h}^{\Gamma}\right)\right)=\tilde{\mathcal{L}}_{h}\left(q_{h}, q_{h}^{\Gamma}\right) \quad \forall\left(q_{h}, q_{h}^{\Gamma}\right) \in V_{h}^{b} \times V_{h}^{\Gamma},
$$

where $\tilde{\mathcal{A}}_{h}$ is obtained from $\mathcal{A}_{h}$ by replacing the bilinear form $\mathcal{A}_{b}^{D G}(\cdot, \cdot)$ with its inconsistent version $\tilde{\mathcal{A}}_{b}^{D G}(\cdot, \cdot)$ and $\tilde{\mathcal{L}}_{h}$ is obtained by replacing the linear operator $\mathcal{L}_{b}^{D G}(\cdot)$ with $\tilde{\mathcal{L}}_{b}^{D G}(\cdot)$. We remark that formulation (23) is no longer consistent due to the discrete nature of the $L^{2}$-projection operator $\Pi_{2}$.

We also remark that well-posedness of the discrete problem (13) is guaranteed if we show that, more in general, problem (23) extended to the space $V^{b}(h) \times V_{h}^{\Gamma}$ is well-posed. The choice of proving this more general property is made for future use in the error analysis.

Taking as a reference $[25,23,4,22]$, we state and prove the following result. Note that for the proof, Assumption 7.1 will play a fundamental role as well as the choice of the discontinuity-penalization parameter $\sigma$.

Lemma 7.4. Let $\sigma: \mathcal{F}_{h} \backslash \Gamma_{h} \rightarrow \mathbb{R}^{+}$be defined as in (17). Then, if Assumption 7.1 holds, the bilinear form $\tilde{\mathcal{A}}_{b}^{D G}(\cdot, \cdot)$ is continuous on $V^{b}(h) \times V^{b}(h)$, and, provided that $\sigma_{0}$ is sufficiently large, it is also coercive on $V^{b}(h) \times V^{b}(h)$, i.e.,

$$
\tilde{\mathcal{A}}_{b}^{D G}(p, q) \lesssim\|q\|_{D G}\|p\|_{D G}, \quad \tilde{\mathcal{A}}_{b}^{D G}(q, q) \gtrsim\|q\|_{D G}^{2},
$$

for any $q, p \in V^{b}(h)$.

Proof. For the proof we follow [22] and [25]. We start with coercivity. For any $q \in V^{b}(h)$,

$$
\begin{aligned}
\tilde{\mathcal{A}}_{b}^{D G}(q, q) & =\|q\|_{D G}^{2}-2 \sum_{F \in \mathcal{F}_{h} \backslash \Gamma_{h}} \int_{F}\left\{\boldsymbol{\nu} \Pi_{2}(\nabla q)\right\} \cdot \llbracket q \rrbracket \\
& =I+I I .
\end{aligned}
$$

Copyright $@$ by SIAM. Unauthorized reproduction of this article is prohibited. 
In order to bound term II, we employ Cauchy-Schwarz's triangular and Young's inequalities to obtain

$$
\begin{aligned}
\sum_{F \in \mathcal{F}_{h} \backslash \Gamma_{h}} \int_{F}\left\{\boldsymbol{\nu} \Pi_{2}(\nabla q)\right\} \cdot \llbracket q \rrbracket \lesssim & \left(\sum_{F \in \mathcal{F}_{h} \backslash \Gamma_{h}}\left\|\sigma_{F}^{-1 / 2} \boldsymbol{\nu}\left(\Pi_{2}\left(\nabla q^{+}\right)+\Pi_{2}\left(\nabla q^{-}\right)\right)\right\|_{L^{2}(F)}^{2}\right)^{1 / 2} \\
& \times\left(\sum_{F \in \mathcal{F}_{h} \backslash \Gamma_{h}}\left\|\sigma_{F}^{1 / 2} \llbracket q \rrbracket\right\|_{L^{2}(F)}^{2}\right)^{1 / 2} \\
\lesssim & \varepsilon \sum_{F \in \mathcal{F}_{h} \backslash \Gamma_{h}}\left(\overline{\boldsymbol{\nu}}_{E^{+}} \sigma_{F}\left\|\Pi_{2}\left(\nabla q^{+}\right)\right\|_{L^{2}(F)}^{2}\right. \\
& \left.+\overline{\boldsymbol{\nu}}_{E^{-}} \sigma_{F}\left\|\Pi_{2}\left(\nabla q^{-}\right)\right\|_{L^{2}(F)}^{2}\right)+\frac{1}{4 \varepsilon}\left\|\sigma_{F}^{1 / 2} \llbracket q \rrbracket\right\|_{L^{2}(F)}^{2} .
\end{aligned}
$$

Employing the inverse inequality (7.2) over the simplices $S_{E}^{F}$ and the definition of the interior penalty parameter $\sigma$, we have

$$
\begin{aligned}
\sum_{F \in \mathcal{F}_{h} \backslash \Gamma_{h}} \int_{F}\left\{\boldsymbol{\nu} \Pi_{2}(\nabla q)\right\} \cdot \llbracket q \rrbracket \lesssim & \frac{\varepsilon}{\sigma_{0}} \sum_{E \in \mathcal{T}_{h}} \sum_{F \in \partial E} \frac{h_{E}|F|}{d\left|S_{E}^{F}\right|}\left\|\Pi_{2}(\nabla q)\right\|_{L^{2}\left(S_{E}^{F}\right)}^{2} \\
& +\frac{1}{4 \varepsilon} \sum_{F \in \mathcal{F}_{h} \backslash \Gamma_{h}}\left\|\sigma_{F}^{1 / 2} \llbracket q \rrbracket\right\|_{L^{2}(F)}^{2} \\
\lesssim & \frac{\varepsilon}{\sigma_{0}} \sum_{E \in \mathcal{T}_{h}}\left\|\boldsymbol{\nu}^{1 / 2} \nabla q\right\|_{L(E)}^{2}+\frac{1}{4 \varepsilon} \sum_{F \in \mathcal{F}_{h} \backslash \Gamma_{h}}\left\|\sigma_{F}^{1 / 2} \llbracket q \rrbracket\right\|_{L^{2}(F)}^{2},
\end{aligned}
$$

where we have used Assumption 7.1 and the bound (19), together with the $L^{2}$-stability of the projector $\Pi_{2}$ and the boundedness of tensor $\boldsymbol{\nu}$. In conclusion, using Assumption 7.1 , we proved that

$$
\tilde{\mathcal{A}}_{b}^{D G}(q, q) \gtrsim\|q\|_{D G}^{2} \quad \text { for all } q \in V^{b}(h)
$$

for an appropriate choice of the constant $\varepsilon$ and for $\sigma_{0}$ large enough. The proof of continuity can be obtained employing analogous arguments.

Lemma 7.5. The bilinear form $\mathcal{A}_{\Gamma}(\cdot, \cdot)$ is coercive and continuous on $V_{h}^{\Gamma} \times V_{h}^{\Gamma}$ with respect to the norm $\|\cdot\|_{\Gamma}$.

Proof. Since $\mathcal{A}_{\Gamma}\left(q_{h}^{\Gamma}, q_{h}^{\Gamma}\right)=\left\|q_{h}^{\Gamma}\right\|_{\Gamma}^{2}$ for any $q_{h}^{\Gamma} \in V_{h}^{\Gamma}, \mathcal{A}_{\Gamma}(\cdot, \cdot)$ is clearly coercive. Continuity follows directly from the Cauchy-Schwarz inequality.

Employing Lemmas 7.4 and 7.5, we can easily prove the well-posedness of the discrete problem (13).

Proof of Theorem 5.2. We have $\mathcal{I}^{D G}\left(\left(q_{h}, q_{h}^{\Gamma}\right),\left(q, q_{h}^{\Gamma}\right)\right)=\left\|\left(q, q_{h}^{\Gamma}\right)\right\|_{\mathcal{I}}^{2}$. Moreover, from Lemmas 7.4 and 7.5 , we know that $\tilde{\mathcal{A}}_{b}^{D G}(q, q) \gtrsim\|q\|_{D G}^{2}$ and $\mathcal{A}_{\Gamma}\left(q_{h}^{\Gamma}, q_{h}^{\Gamma}\right)=\left\|q_{h}^{\Gamma}\right\|_{\Gamma}^{2}$, respectively. Therefore,

$$
\tilde{\mathcal{A}}_{h}\left(\left(q, q_{h}^{\Gamma}\right),\left(q, q_{h}^{\Gamma}\right)\right) \gtrsim\left\|\left(q, q_{h}^{\Gamma}\right)\right\|_{\mathcal{E}_{h}}^{2} \quad \forall\left(q, q_{h}^{\Gamma}\right) \in V^{b}(h) \times V_{h}^{\Gamma} .
$$

Next we prove continuity. Let $\left(q, q_{h}^{\Gamma}\right),\left(w, w_{h}^{\Gamma}\right) \in V^{b}(h) \times V_{h}^{\Gamma}$. Then, from Lemmas 7.4 and 7.5 ,

$$
\begin{aligned}
\tilde{\mathcal{A}}_{b}^{D G}(q, w) & \lesssim\|q\|_{D G}\|w\|_{D G} \lesssim\left\|\left(q, q_{h}^{\Gamma}\right)\right\|_{\mathcal{E}_{h}}\left\|\left(w, w_{h}^{\Gamma}\right)\right\|_{\mathcal{E}_{h}}, \\
\mathcal{A}_{\Gamma}\left(q_{h}^{\Gamma}, w_{h}^{\Gamma}\right) & \lesssim\left\|q_{h}^{\Gamma}\right\|\left\|_{\Gamma}\right\| w_{h}^{\Gamma}\left\|_{\Gamma} \lesssim\right\|\left(q_{h}, q_{h}^{\Gamma}\right)\left\|\left.\right|_{\mathcal{E}_{h}}\right\|\left(w_{h}, w_{h}^{\Gamma}\right) \|_{\mathcal{E}_{h}} .
\end{aligned}
$$

Copyright $\odot$ by SIAM. Unauthorized reproduction of this article is prohibited. 
Finally, from the Cauchy-Schwarz inequality, we get

$$
\begin{aligned}
\mathcal{I}^{D G}\left(\left(q, q_{h}^{\Gamma}\right),\left(w, w_{h}^{\Gamma}\right)\right) \leq & \sum_{F \in \Gamma_{h}}\left\|\beta_{\Gamma}^{1 / 2} \llbracket q \rrbracket\right\|_{L^{2}(F)}^{2}\left\|\beta_{\Gamma}^{1 / 2} \llbracket w \rrbracket\right\|_{L^{2}(F)}^{2} \\
& +\sum_{F \in \Gamma_{h}}\left\|\alpha_{\Gamma}^{1 / 2}\left(\{q\}-q_{h}^{\Gamma}\right)\right\|_{L^{2}(F)}^{2}\left\|\alpha_{\Gamma}^{1 / 2}\left(\{w\}-w_{h}^{\Gamma}\right)\right\|_{L^{2}(F)}^{2} \\
\leq & \left\|\left(q, q_{h}^{\Gamma}\right)\right\|_{\mathcal{E}_{h}}\left\|\left(w, w_{h}^{\Gamma}\right)\right\|_{\mathcal{E}_{h} .}
\end{aligned}
$$

The continuity of $\tilde{\mathcal{L}}_{h}(\cdot)$ on $V^{b}(h) \times V_{h}^{\Gamma}$ can be easily proved using the Cauchy-Schwarz inequality thanks to the regularity assumptions on the forcing terms $f$ and $f_{\Gamma}$.

7.2. Error estimates. In this section we prove that the discrete solution $\left(p_{h}, p_{h}^{\Gamma}\right)$ to problem (13) (or, equivalently, to problem (23)) converges to the exact solution $\left(p, p_{\Gamma}\right)$.

7.2.1. Approximation results. In $[25,23,4]$ standard results on simplices are extended to polytopic elements, considering appropriate coverings and submeshes made of $d$-dimensional simplices (where standard results can be applied) and using appropriate extension operators. In [22] these results are restated in order to be successfully applied also in the case when the number of edges/faces is unbounded. Here we summarize the results contained in [25, 23, 4, 22].

Assumption $7.6([25,23])$. There exists a covering $\mathcal{T}_{\#}$ of $\mathcal{T}_{h}$ (see Definition 5.3) and a positive constant $O_{\Omega}$, independent of the mesh parameters such that

$$
\max _{E \in \mathcal{T}_{h}} O_{E} \leq O_{\Omega}
$$

where, for $E \in \mathcal{T}_{h}, O_{E}=\operatorname{card}\left\{E^{\prime} \in \mathcal{T}_{h}: E^{\prime} \cap T_{E} \neq \emptyset, T_{E} \in \mathcal{T}_{\#}\right.$ s.t. $\left.E \subset T_{E}\right\}$.

Let $\mathscr{E}$ be the classical continuous extension operator, cf. [46]. We can then state the following approximation result.

Lemma 7.7 ([25, 23, 4, 22]). Let $E \in \mathcal{T}_{h}, F \subset \partial E$ denote one of its faces and $T_{E} \in \mathcal{T}_{\#}$ denote the corresponding simplex such that $E \subset T_{E}$ (see Definition 5.3). Suppose that $v \in L^{2}(\Omega)$ is such that $\left.\mathscr{E} v\right|_{T_{E}} \in H^{r_{E}}\left(T_{E}\right)$ for some $r_{E} \geq 0$. Then, if Assumptions 7.1 and 7.6 are satisfied, there exists $\widetilde{\Pi} v$ such that $\left.\widetilde{\Pi} v\right|_{E} \in \mathbb{P}_{k_{E}}(E)$, and the following bound holds:

$$
\|v-\widetilde{\Pi} v\|_{H^{q}(E)} \lesssim \frac{h_{E}^{s_{E}-q}}{k_{E}^{r_{E}-q}}\|\mathscr{E} v\|_{H^{r_{E}\left(T_{E}\right)}}, \quad 0 \leq q \leq r_{E} .
$$

Moreover, if $r_{E}>1 / 2$,

$$
\|v-\widetilde{\Pi} v\|_{L^{2}(\partial E)} \lesssim \frac{h_{E}^{s_{E}-1 / 2}}{k_{E}^{r_{E}-1 / 2}}\|\mathscr{E} v\|_{H^{r_{E}}\left(T_{E}\right)}
$$

Here, $s_{E}=\min \left(k_{E}+1, r_{E}\right)$, and the hidden constants depend on the shape-regularity of $T_{E}$ but are independent of $v, h_{E}, k_{E}$ and the number of faces per element.

Proof. See [25] for a detailed proof of (26) and [22] for the proof of (27).

Note that the fact that estimate (27) holds on the whole boundary $\partial E$ is fundamental for treating the case when the number of faces/edges is not uniformly bounded. 
Finally, for future use, we recall that, using classical interpolation estimates [12], for any $p_{\Gamma} \in H^{r}\left(\Gamma_{h}\right), r \geq 1$, there exists $p_{\Gamma}^{I} \in V_{h}^{\Gamma}$ such that

$$
|| p_{\Gamma}-\left.p_{\Gamma}^{I}\right|_{\Gamma} \lesssim \sum_{F \in \Gamma_{h}} \frac{h_{F}^{s}}{k^{r-1}}\left|p_{\Gamma}\right|_{H^{r}(F)}
$$

with $s=\min \{k+1, r\}$.

7.2.2. Error analysis. Now we have all the ingredients to prove Theorem 5.5. We point out that Galerkin's orthogonality does not hold true due to the inconsistency of the bilinear form $\tilde{\mathcal{A}}_{h}$. Thereby, the error bound will be derived starting from Strang's second lemma. From Proposition 5.2 and Strang's second lemma we directly obtain the following abstract error bound on the error.

Lemma 7.8. Assuming that the hypotheses of Proposition 5.2 are satisfied, it holds that

$$
\begin{aligned}
\left.\left\|\left(p, p_{\Gamma}\right)-\left(p_{h}, p_{h}^{\Gamma}\right)\right\|\right|_{\mathcal{E}_{h}} \lesssim & \inf _{\left(q_{h}, q_{h}^{\Gamma}\right) \in V_{h}^{b} \times V_{h}^{\Gamma}}\left\|\left(p, p_{\Gamma}\right)-\left(q_{h}, q_{h}^{\Gamma}\right)\right\|_{\mathcal{E}_{h}} \\
& +\sup _{\left(w_{h}, w_{h}^{\Gamma}\right) \in V_{h}^{b} \times V_{h}^{\Gamma}} \frac{\left|\mathcal{R}_{h}\left(\left(p, p_{\Gamma}\right),\left(w_{h}, w_{h}^{\Gamma}\right)\right)\right|}{\|\left(w_{h}, w_{h}^{\Gamma}\right)||_{\mathcal{E}_{h}}},
\end{aligned}
$$

where the residual $\mathcal{R}_{h}$ is defined as

$$
\mathcal{R}_{h}\left(\left(p, p_{\Gamma}\right),\left(w_{h}, w_{h}^{\Gamma}\right)\right)=\tilde{\mathcal{A}}_{h}\left(\left(p, p_{\Gamma}\right),\left(w_{h}, w_{h}^{\Gamma}\right)\right)-\mathcal{L}_{h}\left(w_{h}, w_{h}^{\Gamma}\right) .
$$

We can now proceed with the proof of Theorem 5.5.

Proof of Theorem 5.5. From Lemma 7.8 we know that the error satisfies the following bound:

$$
\begin{aligned}
\left\|\left(p, p_{\Gamma}\right)-\left(p_{h}, p_{h}^{\Gamma}\right)\right\|_{\mathcal{E}_{h}} \lesssim \underbrace{\inf _{\left(q_{h}, q_{h}^{\Gamma}\right) \in V_{h}^{b} \times V_{h}^{\Gamma}}\left\|\left(p, p_{\Gamma}\right)-\left(q_{h}, q_{h}^{\Gamma}\right)\right\|_{\mathcal{E}_{h}}}_{I} \\
+\underbrace{\underbrace{}_{\left(w_{h}, w_{h}^{\Gamma}\right) \in V_{h}^{b} \times V_{h}^{\Gamma}} \frac{\left|\mathcal{R}_{h}\left(\left(p, p_{\Gamma}\right),\left(w_{h}, w_{h}^{\Gamma}\right)\right)\right|}{\left\|\left(w_{h}, w_{h}^{\Gamma}\right)\right\|_{\mathcal{E}_{h}}}}_{I I} .
\end{aligned}
$$

We bound the two terms on the right-hand side of (30) separately. We can rewrite term I as

$$
I=\underbrace{\inf _{q_{h} \in V_{h}^{b}}\left\|p-q_{h}\right\|_{D G}^{2}}_{(a)}+\underbrace{\inf _{q_{h}^{\Gamma} \in V_{h}^{\Gamma}}\left\|p_{\Gamma}-q_{h}^{\Gamma}\right\|_{\Gamma}^{2}}_{(b)}+\underbrace{\inf _{\left(q_{h}, q_{h}^{\Gamma}\right) \in V_{h}^{b} \times V_{h}^{\Gamma}}\left\|\left(p-q_{h}, p_{\Gamma}-q_{h}^{\Gamma}\right)\right\|_{\mathcal{I}}^{2}}_{(c)} .
$$

Again we consider each of the three terms separately. To bound term (a), we exploit the two approximation results stated in Lemma 7.7; we obtain that

Copyright $@$ by SIAM. Unauthorized reproduction of this article is prohibited. 


$$
\begin{aligned}
& (a) \leq\|p-\widetilde{\Pi} p\|_{D G}^{2}=\sum_{E \in \mathcal{T}_{h}}\left\|\boldsymbol{\nu}^{1 / 2} \nabla(p-\widetilde{\Pi} p)\right\|_{L^{2}(E)}^{2}+\sum_{F \in \mathcal{F}_{h} \backslash \Gamma_{h}} \sigma_{F}\|\llbracket p-\widetilde{\Pi} p \rrbracket\|_{L^{2}(F)}^{2} \\
& \lesssim \sum_{E \in \mathcal{T}_{h}}\left[\overline{\boldsymbol{\nu}}_{E}|p-\widetilde{\Pi} p|_{H^{1}(E)}^{2}+\left(\max _{F \subset \partial E \backslash \Gamma} \sigma_{F}\right)\|p-\widetilde{\Pi} p\|_{L^{2}(\partial E \backslash \Gamma)}^{2}\right] \\
& \lesssim \sum_{E \in \mathcal{T}_{h}}\left[\frac{h_{E}^{2\left(s_{E}-1\right)}}{k_{E}^{2\left(r_{E}-1\right)}} \overline{\boldsymbol{\nu}}_{E}\|\mathscr{E} p\|_{H^{r_{E}\left(T_{E}\right)}}^{2}+\frac{h_{E}^{2\left(s_{E}-1 / 2\right)}}{k_{E}^{2\left(r_{E}-1 / 2\right)}}\left(\max _{F \subset \partial E \backslash \Gamma} \sigma_{F}\right)\|\mathscr{E} p\|_{H^{r_{E}\left(T_{E}\right)}}^{2}\right] \\
& =\sum_{E \in \mathcal{T}_{h}} \frac{h_{E}^{2\left(s_{E}-1\right)}}{k_{E}^{2\left(r_{E}-1\right)}}\|\mathscr{E} p\|_{H^{r_{E}\left(T_{E}\right)}}^{2}\left(\overline{\boldsymbol{\nu}}_{E}+\frac{h_{E}}{k_{E}}\left(\max _{F \subset \partial E \backslash \Gamma} \sigma_{F}\right)\right) .
\end{aligned}
$$

Using classical interpolation estimates (see (28)) we can bound term (b) as follows:

$$
(b) \leq\left\|p_{\Gamma}-p_{\Gamma}^{I}\right\|_{\Gamma}^{2} \lesssim \sum_{F \in \Gamma_{h}} \frac{h_{F}^{2 k}}{k^{2(r-1)}}\left|p_{\Gamma}\right|_{H^{r}(F)}^{2} .
$$

Finally, for term (c), we have

$$
\begin{array}{r}
(c) \leq\left\|\left(p-\widetilde{\Pi} p, p_{\Gamma}-p_{\Gamma}^{I}\right)\right\|_{\mathcal{I}}^{2} \lesssim \beta_{\Gamma} \sum_{F \in \Gamma_{h}}\|\llbracket p-\widetilde{\Pi} p \rrbracket\|_{L^{2}(F)}^{2}+\alpha_{\Gamma} \sum_{F \in \Gamma_{h}}\|\{p-\widetilde{\Pi} p\}\|_{L^{2}(F)}^{2} \\
+\alpha_{\Gamma} \sum_{F \in \Gamma_{h}}\left\|p_{\Gamma}-p_{\Gamma}^{I}\right\|_{L^{2}(F)}^{2} .
\end{array}
$$

Exploiting the approximation result (27), we obtain

$$
\begin{aligned}
\beta_{\Gamma} \sum_{F \in \Gamma_{h}}\|\llbracket p-\widetilde{\Pi} p \rrbracket\|_{L^{2}(F)}^{2} \leq \beta_{\Gamma} \sum_{\substack{E \in \mathcal{T}_{h} \\
\partial E \cap \Gamma \neq \emptyset}}\|p-\widetilde{\Pi} p\|_{L^{2}(\partial E)}^{2} \lesssim \beta_{\Gamma} \sum_{\substack{E \in \mathcal{T}_{h} \\
\partial E \cap \Gamma \neq \emptyset}} \frac{h_{E}^{2\left(s_{E}-\frac{1}{2}\right)}}{k_{E}^{2\left(r_{E}-\frac{1}{2}\right)}}\|\mathscr{E} p\|_{H^{r_{E}}\left(T_{E}\right)}^{2} \\
=\beta_{\Gamma} \sum_{\substack{E \in \mathcal{T}_{h} \\
\partial E \cap \Gamma \neq \emptyset}} \frac{h_{E}^{2\left(s_{E}-1\right)}}{k_{E}^{2\left(r_{E}-1\right)}}\|\mathscr{E} p\|_{H^{r_{E}}\left(T_{E}\right)}^{2} \frac{h_{E}}{k_{E}}
\end{aligned}
$$

Similarly, we have

$$
\alpha_{\Gamma} \sum_{F \in \Gamma_{h}}\|\{p-\widetilde{\Pi} p\}\|_{L^{2}(F)}^{2} \lesssim \alpha_{\Gamma} \sum_{\substack{E \in \mathcal{T}_{h} \\ \partial E \cap \Gamma \neq \emptyset}} \frac{h_{E}^{2\left(s_{E}-1\right)}}{k_{E}^{2\left(r_{E}-1\right)}} \frac{h_{E}}{k_{E}}\|\mathscr{E} p\|_{H^{r_{E}}\left(T_{E}\right)}^{2} .
$$

Finally, using again classical interpolation estimates, we deduce that

$$
\alpha_{\Gamma} \sum_{F \in \Gamma_{h}}\left\|p_{\Gamma}-p_{\Gamma}^{I}\right\|_{L^{2}(F)}^{2} \lesssim \alpha_{\Gamma} \sum_{F \in \Gamma_{h}} \frac{h_{F}^{2 k}}{k^{2(r-1)}}\left|p_{\Gamma}\right|_{H^{r}(F)}^{2} .
$$

In conclusion, combining all the previous estimates, we can bound the term I on the right-hand side of (30) as follows:

$$
\begin{aligned}
& I \lesssim \sum_{F \in \Gamma_{h}} h_{F}^{2 k}\left|p_{\Gamma}\right|_{H^{k+1}(F)}^{2} \\
& +\sum_{E \in \mathcal{T}_{h}} \frac{h_{E}^{2\left(s_{E}-1\right)}}{k_{E}^{2\left(r_{E}-1\right)}}\|\mathscr{E} p\|_{H^{r_{E}\left(T_{E}\right)}}^{2}\left[\overline{\boldsymbol{\nu}}_{E}+h_{E} k_{E}^{-1}\left(\max _{F \subset \partial E \backslash \Gamma} \sigma_{F}\right)+\left(\alpha_{\Gamma}+\beta_{\Gamma}\right) h_{E} k_{E}^{-1}\right] .
\end{aligned}
$$

Copyright $@$ by SIAM. Unauthorized reproduction of this article is prohibited. 
Next, we derive a bound on the term II on the right-hand side of (30). First, we note that integrating by parts elementwise and using that the couple $\left(p, p_{\Gamma}\right)$ satisfies $(9)$ as well as the regularity assumption 5.4 , we can rewrite the residual $\mathcal{R}_{h}$ as

$$
\mathcal{R}_{h}\left(\left(p, p_{\Gamma}\right),\left(w_{h}, w_{h}^{\Gamma}\right)\right)=\sum_{F \in \mathcal{F}_{h} \backslash \Gamma_{h}} \int_{F}\left\{\boldsymbol{\nu}\left(\nabla p-\Pi_{2}(\nabla p)\right)\right\} \cdot \llbracket w_{h} \rrbracket .
$$

Employing the Cauchy-Schwarz inequality and the definition of the norm $\|\cdot\|_{\mathcal{E}_{h}}$, we then obtain

$$
I I \leq\left(\sum_{F \in \mathcal{F}_{h} \backslash \Gamma_{h}} \sigma_{F}^{-1} \int_{F}\left|\left\{\boldsymbol{\nu}\left(\nabla p-\Pi_{2}(\nabla p)\right)\right\}\right|^{2}\right)^{1 / 2} .
$$

If we still denote by $\widetilde{\Pi}$ the vector-valued generalization of the projection operator $\widetilde{\Pi}$ defined in Lemma 7.7, we observe that

$$
\begin{aligned}
\sum_{F \in \mathcal{F}_{h} \backslash \Gamma_{h}} \sigma_{F}^{-1} \int_{F}\left|\left\{\boldsymbol{\nu}\left(\nabla p-\Pi_{2}(\nabla p)\right)\right\}\right|^{2} & \lesssim \sum_{F \in \mathcal{F}_{h} \backslash \Gamma_{h}} \sigma_{F}^{-1} \int_{F}|\{\boldsymbol{\nu}(\nabla p-\widetilde{\Pi}(\nabla p))\}|^{2} \\
& +\sum_{F \in \mathcal{F}_{h} \backslash \Gamma_{h}} \sigma_{F}^{-1} \int_{F}\left|\left\{\boldsymbol{\nu} \Pi_{2}(\nabla p-\widetilde{\Pi}(\nabla p))\right\}\right|^{2} \\
& \equiv(1)+(2) .
\end{aligned}
$$

To bound term (1), we proceed as above, employing the approximation result stated in Lemma 7.7. We obtain

$$
(1) \lesssim \sum_{E \in \mathcal{T}_{h}} \frac{h_{E}^{2\left(s_{E}-1\right)}}{k_{E}^{2\left(r_{E}-1\right)}}\left(\overline{\boldsymbol{\nu}}_{E}\left(\max _{F \subset \partial E \backslash \Gamma} \sigma_{F}^{-1}\right) \frac{h_{E}^{-1}}{k_{E}^{-1}}\right)\|\mathscr{E} p\|_{H^{r_{E}}\left(T_{E}\right)}^{2} .
$$

Exploiting, in order, the boundedness of the permeability tensor $\boldsymbol{\nu}$, the inverse inequality (22), the $L^{2}$-stability of the projector $\Pi_{2}$, and the approximation results stated in Lemma 7.7, we can bound term (2) as follows:

$$
\begin{aligned}
(2) & \lesssim \sum_{E \in \mathcal{T}_{h}}\left(\max _{F \subset \partial E \backslash \Gamma} \sigma_{F}^{-1}\right) \overline{\boldsymbol{\nu}}_{E}\left\|\Pi_{2}(\widetilde{\Pi}(\nabla p)-\nabla p)\right\|_{L^{2}(\partial E \backslash \Gamma)}^{2} \\
& \lesssim \sum_{E \in \mathcal{T}_{h}}\left(\max _{F \subset \partial E \backslash \Gamma} \sigma_{F}^{-1}\right) \overline{\boldsymbol{\nu}}_{E} \frac{k_{E}^{2}}{h_{E}}\|\widetilde{\Pi}(\nabla p)-\nabla p\|_{L^{2}(E)}^{2} \\
& \lesssim \sum_{E \in \mathcal{T}_{h}} \frac{h_{E}^{2\left(s_{E}-1\right)}}{k_{E}^{2\left(r_{E}-1\right)}}\|\mathscr{E} p\|_{H^{r_{E}}\left(T_{E}\right)}^{2}\left(\overline{\boldsymbol{\nu}}_{E} \frac{k_{E}^{2}}{h_{E}}\left(\max _{F \subset \partial E \backslash \Gamma} \sigma_{F}^{-1}\right)\right) .
\end{aligned}
$$

Hence, term II on the right-hand side of (30) may be bounded as

$$
\begin{aligned}
I I \lesssim \sum_{E \in \mathcal{T}_{h}} \frac{h_{E}^{2\left(s_{E}-1\right)}}{k_{E}^{2\left(r_{E}-1\right)}}\|\mathscr{E} p\|_{H^{r_{E}\left(T_{E}\right)}}^{2}\left[\overline{\boldsymbol{\nu}}_{E}\left(\max _{F \subset \partial E \backslash \Gamma} \sigma_{F}^{-1}\right) h_{E}^{-1} k_{E}\right. & \\
& \left.+\overline{\boldsymbol{\nu}}_{E}\left(\max _{F \subset \partial E \backslash \Gamma} \sigma_{F}^{-1}\right) h_{E}^{-1} k_{E}^{2}\right] .
\end{aligned}
$$

Finally, substituting (31) and (32) into (30) leads to the thesis. 
8. Concluding remarks. In this work we have presented a DG approximation on polytopic grids of Darcy's flow through a fractured porous medium. For the analysis we have assumed the medium to be cut by a single nonimmersed fracture, and we have obtained a priori error estimates. These estimates have been validated through numerical tests. Moreover, we have shown that our approach is also robust in more complex geometric configurations, performing numerical experiments involving networks of partially immersed fractures and networks of intersecting fractures.

Future extensions include the analysis of the case of a network of (partially immersed) fractures intersecting each other. As already pointed out in section 6.6, to deal with this case, we need to complete our model with suitable physical conditions at the intersection points, imposing, for example, the continuity of pressure and flux conservation as in $[45,18]$. From the DG-discretization point of view, the key point is the generalization of the concepts of jump and average at the intersection points, similarly to what has been done for linear DG approximation of elliptic PDEs on surfaces in [30] and then extended to high order in [5]. The aforementioned works will also be taken as a reference for an extension of our numerical model to the case of curved fractures.

Acknowledgments. We thank the anonymous reviewers for their very careful reading of the paper. We believe that their many useful criticisms and suggestions contributed to improve substantially the quality and readability of our paper. We also want to thank A. Scotti for useful discussion and remarks.

\section{REFERENCES}

[1] C. Alboin, J. Jaffré, J. E. Roberts, and C. Serres, Modeling Fractures as Interfaces for Flow and Transport in Porous Media, in Fluid Flow and Transport in Porous Media: Mathematical and Numerical Treatment (South Hadley, MA, 2001), Contemp. Math., Amer. Math. Soc. 295, American Mathematical Society, Providence, RI, 2002, pp. 13-24.

[2] C. Alboin, J. Jaffré, J. E. Roberts, X. Wang, and C. Serres, Domain Decomposition for Some Transmission Problems in Flow in Porous Media, in Numerical Treatment of Multiphase Flows in Porous Media, Lecture Notes in Phys. 552, Springer, Berlin, 2000, pp. 22-34.

[3] P. Angot, F. Boyer, And F. Hubert, Asymptotic and numerical modelling of flows in fractured porous media, M2AN Math. Model. Numer. Anal., 43 (2009), pp. 239-275.

[4] P. F. Antonietti, A. Cangiani, J. Collis, Z. Dong, E. H. Georgoulis, S. Giani, and P. Houston, Review of Discontinuous Galerkin Finite Element Methods for Partial Differential Equations on Complicated Domains, Lecture Notes in Computational Science and Engineering, 2016.

[5] P. F. Antonietti, A. Dedner, P. Madhavan, S. Stangalino, B. Stinner, and M. Verani, High order discontinuous Galerkin methods for elliptic problems on surfaces, SIAM J. Numer. Anal., 53 (2015), pp. 1145-1171.

[6] P. F. Antonietti, L. Formaggia, A. Scotti, M. Verani, and N. Verzotti, Mimetic finite difference approximation of flows in fractured porous media, M2AN Math. Model. Numer. Anal., 50 (2016), pp. 809-832.

[7] P. F. Antonietti, S. Giani, And P. Houston, hp-version composite discontinuous Galerkin methods for elliptic problems on complicated domains, SIAM J. Sci. Comput., 35 (2013), pp. A1417-A1439.

[8] P. F. Antonietti, S. Giani, and P. Houston, Domain decomposition preconditioners for discontinuous Galerkin methods for elliptic problems on complicated domains, J. Sci. Comput., 60 (2014), pp. 203-227.

[9] P. F. Antonietti, P. Houston, X. Hu, M. Sarti, and M. Verani, Multigrid algorithms for hp-version interior penalty discontinuous Galerkin methods on polygonal and polyhedral meshes, Calcolo, 54 (2017), pp. 1169-1198.

[10] D. N. ARNOLD, An interior penalty finite element method with discontinuous elements, SIAM J. Numer. Anal., 19 (1982), pp. 742-760. 
[11] D. N. Arnold, F. Brezzi, B. Cockburn, And L. D. Marini, Unified analysis of discontinuous Galerkin methods for elliptic problems, SIAM J. Numer. Anal., 39 (2001/2002), pp. 17491779.

[12] I. BABUŠKA AND M. SURI, The $h p$ version of the finite element method with quasi-uniform meshes, RAIRO Model. Math. Anal. Numer., 21 (1987), pp. 199-238.

[13] G. A. BAKER, Finite element methods for elliptic equations using nonconforming elements, Math. Comp., 31 (1977), pp. 45-59.

[14] F. BASSI AND S. ReBAY, A high-order accurate discontinuous finite element method for the numerical solution of the compressible Navier-Stokes equations, J. Comput. Phys., 131 (1997), pp. 267-279.

[15] J. Bear, C. F. Tsang, and G. D. Marsily, Flow and Contaminant Transport in Fractured Rocks, Academic Press, San Diego, CA, 1993.

[16] L. Beirão da Veiga, F. Brezzi, A. Cangiani, G. Manzini, L. D. Marini, and A. Russo, Basic principles of virtual element methods, Math. Models Methods Appl. Sci., 23 (2013), pp. 199-214.

[17] L. Beirão Da Veiga, F. Brezzi, L. Marini, And A. Russo, Virtual element method for general second-order elliptic problems on polygonal meshes, Math. Models Methods Appl. Sci., 26 (2016), pp. 729-750.

[18] M. F. Benedetto, S. Berrone, And S. Scialò, A globally conforming method for solving flow in discrete fracture networks using the virtual element method, Finite Elem. Anal. Des., 109 (2016), pp. 23-36.

[19] S. Berrone, S. Pieraccini, and S. Scialò, A PDE-constrained optimization formulation for discrete fracture network flows, SIAM J. Sci. Comput., 35 (2013), pp. B487-B510.

[20] S. Berrone, S. Pieraccini, And S. Scialò, An optimization approach for large scale simulations of discrete fracture network flows, J. Comput. Phys., 256 (2014), pp. 838-853.

[21] F. BrezzI, B. Cockburn, L. D. Marini, And E. Süli, Stabilization mechanisms in discontinuous Galerkin finite element methods, Comput. Methods Appl. Mech. Engrg., 195 (2006), pp. 3293-3310.

[22] A. Cangiani, Z. Dong, and E. H. Georgoulis, hp-version space-time discontinuous Galerkin methods for parabolic problems on prismatic meshes, SIAM J. Sci. Comp., 39 (2017), pp. A1251-A1279.

[23] A. Cangiani, Z. Dong, E. H. Georgoulis, and P. Houston, hp-version discontinuous Galerkin methods for advection-diffusion-reaction problems on polytopic meshes, ESAIM Math. Model. Numer. Anal., 50 (2016), pp. 699-725.

[24] A. Cangiani, Z. Dong, E. H. Georgoulis, and P. Houston, $h p$-Version Discontinuous Galerkin Methods on Polytopic Meshes, Springer Briefs in Mathematics, Springer International Publishing, Berlin, 2017.

[25] A. Cangiani, E. H. Georgoulis, and P. Houston, hp-version discontinuous Galerkin methods on polygonal and polyhedral meshes, Math. Models Methods Appl. Sci., 24 (2014), pp. 2009-2041.

[26] P. Castillo, B. Cockburn, I. Perugia, and D. SchÖtzau, An a priori error analysis of the local discontinuous Galerkin method for elliptic problems, SIAM J. Numer. Anal., 38 (2000), pp. 1676-1706.

[27] F. A. Chave, D. Di Pietro, and L. Formaggia, A Hybrid High-Order Method for Darcy Flows in Fractured Porous Media, HAL ID hal-01482925, 2017.

[28] B. Cockburn and C. Dawson, Some Extensions of the Local Discontinuous Galerkin Method for Convection-Diffusion Equations in Multidimensions, in The Mathematics of Finite Elements and Applications, X, MAFELAP 1999 (Uxbridge), Elsevier, Oxford, 2000, pp. 225238.

[29] C. D'Angelo And A. Scotti, A mixed finite element method for Darcy flow in fractured porous media with non-matching grids, ESAIM Math. Model. Numer. Anal., 46 (2012), pp. $465-489$.

[30] A. Dedner, P. Madhavan, And B. Stinner, Analysis of the discontinuous Galerkin method for elliptic problems on surfaces, IMA J. Numer. Anal., 33 (2013), pp. 952-973.

[31] D. A. Di Pietro And A. Ern, Mathematical Aspects of Discontinuous Galerkin Methods, Vol. 69, Springer Science \& Business Media, Berlin, 2011.

[32] D. A. Di Pietro And A. Ern, A hybrid high-order locking-free method for linear elasticity on general meshes, Comput. Methods Appl. Mech. Engrg., 283 (2015), pp. 1-21.

[33] D. A. Di Pietro, A. ERn, And S. Lemaire, An arbitrary-order and compact-stencil discretization of diffusion on general meshes based on local reconstruction operators., Comput. Methods Appl. Math., 14 (2014), pp. 461-472.

Copyright (c) by SIAM. Unauthorized reproduction of this article is prohibited. 
[34] J. Dolbow, N. Mö̈s, And T. Belytschko, An extended finite element method for modeling crack growth with frictional contact, Comput. Methods Appl. Mech. Engrg., 190 (2001), pp. 6825-6846.

[35] J. Douglas, JR., And T. Dupont, Interior Penalty Procedures for Elliptic and Parabolic Galerkin Methods, in Computing Methods in Applied Sciences (Second Internat. Sympos., Lecture Notes in Phys., Vol. 58, Versailles, 1975), Springer, Berlin, 1976, pp. 207-216.

[36] T.-P. Fries And T. Belytschko, The extended/generalized finite element method: An overview of the method and its applications, Internat. J. Numer. Methods Engrg., 84 (2010), pp. 253-304.

[37] N. Frih, J. E. Roberts, And A. SAAdA, Modeling fractures as interfaces: A model for Forchheimer fractures, Comput. Geosci., 12 (2008), pp. 91-104.

[38] A. Fumagalli And A. Scotti, A numerical method for two-phase flow in fractured porous media with non-matching grids, Adv. Water Resources, 62, Part C (2013), pp. 454-464.

[39] A. Fumagalli and A. Scotti, An efficient xfem approximation of Darcy flows in arbitrarily fractured porous media, Oil Gas Sci. Technol. Rev. IFP Energies nouvelles, 69 (2014), pp. 555-564.

[40] J. S. Hesthaven And T. Warburton, Nodal Discontinuous Galerkin Methods: Algorithms, Analysis, and Applications, Springer Science \& Business Media, Berlin, 2007.

[41] J. Jaffrré, M. MnejJa, And J. Roberts, A discrete fracture model for two-phase flow with matrix-fracture interaction, Proc. Comput. Sci., 4 (2011), pp. 967-973.

[42] V. Martin, J. Jaffré, And J. E. Roberts, Modeling fractures and barriers as interfaces for flow in porous media, SIAM J. Sci. Comput., 26 (2005), pp. 1667-1691.

[43] W. Reed and T. Hill, Triangular Mesh Methods for the nEutron Transport Equation, Los Alamos Report LA-UR-73-479, 1973.

[44] B. RiviÈre, Discontinuous Galerkin Methods for Solving Elliptic and Parabolic Equations, Frontiers in Applied Mathematics 35, SIAM, Philadelphia, PA, 2008.

[45] A. Scotti, L. Formaggia, and F. Sottocasa, Analysis of a mimetic finite difference approximation of flows in fractured porous media, ESAIM. Math. Model. Numer. Anal., (2017).

[46] E. M. Stein, Singular Integrals and Differentiability Properties of Functions, Vol. 2, Princeton University Press, Princeton, NJ, 1970.

[47] C. Talischi, G. H. Paulino, A. Pereira, and I. F. Menezes, Polymesher: A general-purpose mesh generator for polygonal elements written in MATLAB, Struct. Multidiscip. Optim., 45 (2012), pp. 309-328.

[48] T. Warburton and J. S. Hesthaven, On the constants in hp-finite element trace inverse inequalities, Comput. Methods Appl. Mech. Engrg., 192 (2003), pp. 2765-2773.

[49] M. F. WheELer, An elliptic collocation-finite element method with interior penalties, SIAM J. Numer. Anal., 15 (1978), pp. 152-161.

Copyright (c) by SIAM. Unauthorized reproduction of this article is prohibited. 